ARTICLE

DOI: $10.1038 / s 41467-017-02332-3$

\title{
Small-molecule TFEB pathway agonists that ameliorate metabolic syndrome in mice and extend C. elegans lifespan
}

Chensu Wang ${ }^{1,2}$, Hanspeter Niederstrasser ${ }^{3}$, Peter M. Douglas ${ }^{4}$, Rueyling Lin ${ }^{4}$, Juan Jaramillo ${ }^{4}$, Yang Li ${ }^{1}$, Nathaniel W. Oswald ${ }^{3}$, Anwu Zhou ${ }^{3}$, Elizabeth A. McMillan ${ }^{2}$, Saurabh Mendiratta ${ }^{2}$, Zhaohui Wang ${ }^{1}$, Tian Zhao', Zhiqaing Lin', Min Luo ${ }^{1}$, Gang Huang ${ }^{1}$, Rolf A. Brekken¹,5, Bruce A. Posner ${ }^{3}$, John B. MacMillan ${ }^{3}$, Jinming $\mathrm{Gao}^{1} \&$ Michael A. White ${ }^{2}$

Drugs that mirror the cellular effects of starvation mimics are considered promising therapeutics for common metabolic disorders, such as obesity, liver steatosis, and for ageing. Starvation, or caloric restriction, is known to activate the transcription factor EB (TFEB), a master regulator of lipid metabolism and lysosomal biogenesis and function. Here, we report a nanotechnology-enabled high-throughput screen to identify small-molecule agonists of TFEB and discover three novel compounds that promote autophagolysosomal activity. The three lead compounds include the clinically approved drug, digoxin; the marine-derived natural product, ikarugamycin; and the synthetic compound, alexidine dihydrochloride, which is known to act on a mitochondrial target. Mode of action studies reveal that these compounds activate TFEB via three distinct $\mathrm{Ca}^{2+}$-dependent mechanisms. Formulation of these compounds in liver-tropic biodegradable, biocompatible nanoparticles confers hepatoprotection against diet-induced steatosis in murine models and extends lifespan of Caenorhabditis elegans. These results support the therapeutic potential of small-molecule TFEB activators for the treatment of metabolic and age-related disorders.

\footnotetext{
${ }^{1}$ Department of Pharmacology, Simmons Comprehensive Cancer Center, University of Texas Southwestern Medical Center, 5323 Harry Hines Boulevard, Dallas, TX 75390, USA. ${ }^{2}$ Department of Cell Biology, University of Texas Southwestern Medical Center, 5323 Harry Hines Boulevard, Dallas, TX 75390 , USA. ${ }^{3}$ Department of Biochemistry, University of Texas Southwestern Medical Center, 5323 Harry Hines Boulevard, Dallas, TX 75390, USA. ${ }^{4}$ Department of Molecular Biology, University of Texas Southwestern Medical Center, 5323 Harry Hines Boulevard, Dallas, TX 75390, USA. ${ }^{5}$ Department of Surgery, University of Texas Southwestern Medical Center, 5323 Harry Hines Boulevard, Dallas, TX 75390, USA. Correspondence and requests for materials should be addressed to J.G. (email: jinming.gao@utsouthwestern.edu) or to M.A.W. (email: michael.white@utsouthwestern.edu)
} 
A utophagosome-lysosome biogenesis is a major adaptive catabolic process that both generates nutrients and energy during starvation and maintains homeostasis under nutrient-rich conditions. Impairment of this process is mechanistically associated with metabolic disorders and ageing. In metabolic syndromes such as obesity ${ }^{1,2}$ and fatty liver disease $e^{2,3}$, excess nutrients increase demand for degradative autophagylysosome machinery and challenge the adaptive response capacity. Ineffective digestion of macromolecules (lipids, proteins, and glycogen) and impaired organelle turnover compromise metabolic activity at the tissue level, provoke intracellular stresses, and exacerbate collateral defects in insulin action or other metabolic pathologies. During ageing and within age-related disorders ${ }^{4-6}$, a steady decline in productive autophagy impairs clearance of defective organelles leading pathological accumulation of proapoptotic factors and reactive oxygen species (ROS). Therefore, pharmacological interventions that enhance lysosome function are emerging as a promising strategy to ameliorate metabolic symptoms and promote longevity.

The transcription factor EB (TFEB) positively modulates lipid catabolism $^{7}$ and promotes longevity ${ }^{8}$. This is a consequence of direct induction of the "coordinated lysosomal expression and regulation" (CLEAR) network ${ }^{9}$, which includes genes that control autophagy, lysosome biogenesis, and lipolysis ${ }^{7}$, 10-13. TFEB belongs to microphthalmia-associated transcription factor (MITF)/transcriptional factor E (TFE) family (MiT) of basic helix-loop-helix leucine zipper transcriptional factors that includes MITF, TFEB, transcription factor E3 (TFE3) and transcription factor EC (TFEC) ${ }^{14-16}$. TFEB and TFE3 share extensively overlapping functions and regulatory mechanisms ${ }^{10}, 17-19$. Notably, TFEB/TFE3 overexpression in the liver is sufficient to mimic many transcriptional changes that occur during starvation $^{7,20}$. In Caenorhabditis elegans (C. elegans), overexpression of the TFEB homolog HLH-30 also increases lifespan, likely through induction of macroautophagy ${ }^{8}$. Consequently, TFEB/TFE3 agonists are of interest for potential therapeutic intervention for some metabolic disorders and/or ageing.

Here, we establish a nanotechnology-enabled screening strategy to identify small-molecule TFEB agonists that shift maturation of autophagosomes to degradative autolysosomes. From a chemical library of approximately 15,000 compounds, we identified a small cohort of FDA-approved drugs, marine-derived natural product fractions and synthetic small molecules that promote lysosomal maturation via TFEB activation. Mechanism of action and preclinical proof of concept studies were completed for three molecules: digoxin, ikarugamycin, and alexidine

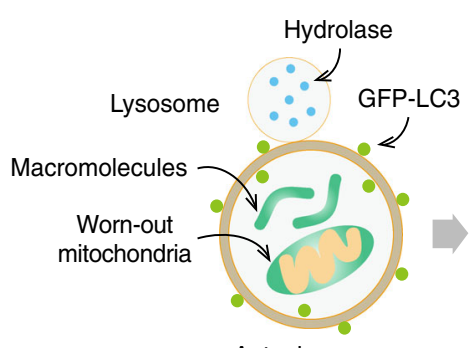

Overall GFP signal

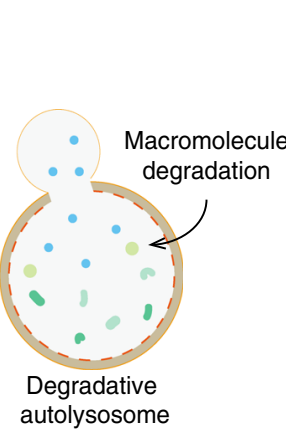

autolysosome
Normal
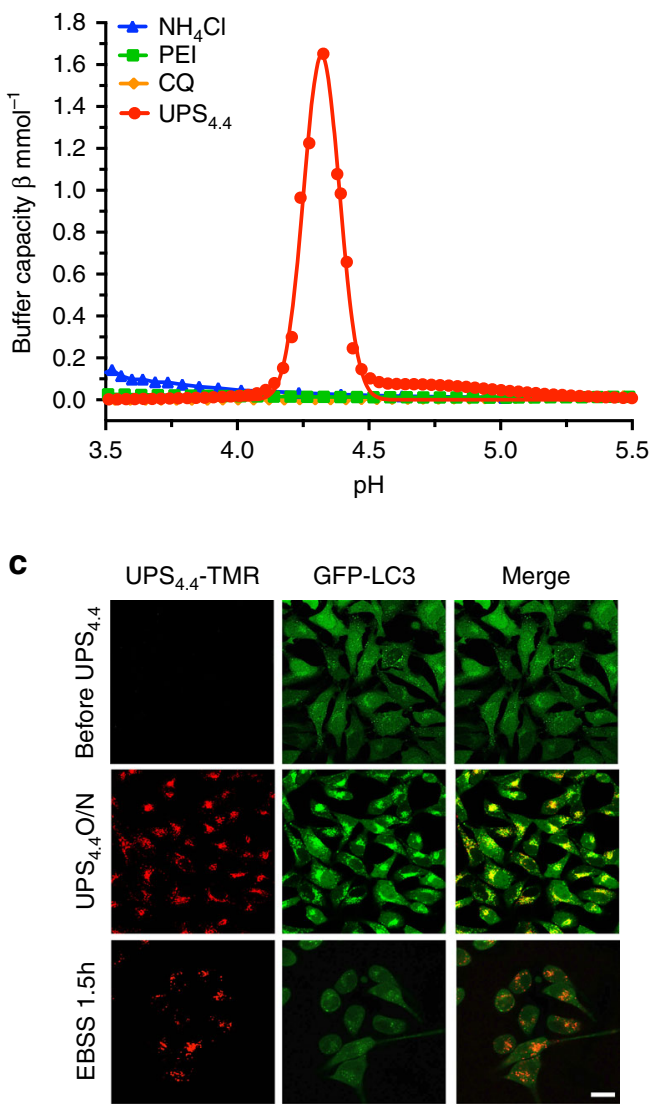

Fig. 1 A UPS-enabled compound screen design that allows for discovery of agents that promote maturation of autophagosomes to degradative autolysosomes. a A schematic showing the design of a quantitative high-throughput cell-based assay for agents that promote maturation of autophagosomes to degradative autolysosomes. Under normal conditions, GFP-LC3 is degraded in autolysosomes resulting in a mild decrease in total fluorescent intensity of GFP signals. UPS 4.4 -buffered autolysosomes have a pH environment that is not optimal for hydrolase activation. In consequence, GFP-LC3 accumulates in the cytosol resulting in a detectable increase in GFP fluorescence intensity as compared to controls. Compounds that promote autophagic flux and lysosomal function overcome the buffering effect of UPS 4.4 and transition the accumulated defective autolysosomes into degradative autolysosomes. This results in a reduction in GFP fluorescence intensity that is reproducibly detectable in a high-throughput setting. $\mathbf{b}$ Buffer capacity ( $\beta$ ) of $\mathrm{UPS}_{4.4}, \mathrm{NH}_{4} \mathrm{Cl}$, chloroquine (CQ) and polyethylenimine (PEI) was plotted as a function of $\mathrm{pH}$. c Representative images showing the effect of UPS $4.4^{-} \mathrm{TMR}$ treatment and a subsequent nutrient-starvation on GFP-LC3 puncta accumulation. Scale bar, $20 \mu \mathrm{m}$ 
dihydrochloride, which engaged TFEB activation mechanisms via three distinct $\mathrm{Ca}^{2+}$ sources and $\mathrm{Ca}^{2+}$-sensing pathways. All three compounds improved lipid metabolism and overcame insulinresistance in a mouse model of diet-induced fatty liver disease. Furthermore, ikarugamycin was delivered to nematode cultures, and resulted in activation of the nematode TFEB ortholog and lifespan extension. These molecules represent leads for development of therapeutic strategies directed against metabolic syndromes, ageing, and age-related disorders, and can serve as pharmacological tools to help provide new biological insights on $\mathrm{Ca}^{2+}$-dependent regulation of cell regulatory systems that impact lysosomes/autophagosome functions.

\section{Results}

A UPS-enabled high-throughput screen for TFEB agonists. A key functional consequence of TFEB activation is enhanced clearance of deleterious macromolecules and organelles through autophagic and lysosomal degradation. Therefore, in order to identify new chemical probes that promote TFEB activity, we first designed a quantitative high-throughput cell-based assay for agents that promote maturation of autophagosomes to degradative autolysosomes (Fig. 1a). This was enabled by a fine-scale UPS nanobuffer library ${ }^{21}$, where each micelle nanoparticle is composed of $\sim 800$ copolymer chains with a total of 60,000 ionizable tertiary amine groups ${ }^{22}$. At specific transition $\mathrm{pH}$, each micelle undergoes a phase transition, or de-micellization, which renders a strong buffering capacity within $0.3 \mathrm{pH}$ range. This unique $\mathrm{pH}$ cooperative buffer effect was implemented to clamp the luminal $\mathrm{pH}$ of endocytic organelles at distinct maturation stages ${ }^{23}$.
Among the UPS nanoprobes, UPS $_{4.4}$ specifically arrests lysosomal acidification at $\mathrm{pH} \sim 4.4$ (Fig. $1 \mathrm{~b}$ and Supplementary Fig. 1a), thereby inhibiting lysosomal/autolysosomal hydrolysis of macromolecules without inhibiting the regulation of mammalian target of rapamycin complex 1 (mTORC1) on lysosomes ${ }^{23}$. Turnover of microtubule-associated protein 1A/1B light chain 3 (LC3), the ortholog of yeast autophagy-related protein 8 (ATG8), was selected as a quantitative measure of lysosome maturation. LC3-II (lipid-modified form of LC3) coats the double membrane structures that encapsulate material that is delivered to autolysosomes, and is itself degraded within those compartments ${ }^{24}$. Thus, GFP-LC3 fusion proteins are commonly employed as livecell markers for monitoring autophagic flux. UPS $_{4.4}$ exposure was sufficient to induce a remarkable accumulation of cytoplasmic GFP-LC3 puncta (Fig. 1c and Supplementary Fig. 1b) that colocalized with fluorescently labeled UPS $_{4.4}$ nanoprobes as well as lysosomal marker LAMP1 (UPS ${ }_{4.4}$-TMR, Supplementary Fig. 1c, d). Moreover, nutrient restriction was sufficient to promote vacuolar ATPase-dependent clearance of these puncta within 90 min, with consequent reduction of GFP fluorescence intensity (Fig. 1c and Supplementary Fig. 1e, f), resulting in an almost binary ON/OFF signal that can be accurately measured by a microplate reader in a high-throughput screen setting. Blocking autolysosomal functions by $\mathrm{UPS}_{4.4}$ resulted in a considerable increase in GFP-LC3 puncta accumulation and fluorescence intensity (six-fold) over starvation-induced autophagic degradation. This is in contrast to a maximal 1.5 -fold fed versus starvation signal in the absence of $\mathrm{UPS}_{4.4}$ (Supplementary Fig. 1g, h). The ready clearance of $\mathrm{UPS}_{4.4}$-induced accumulation of GFPLC3 indicated the presence of a dynamic cell biological system,

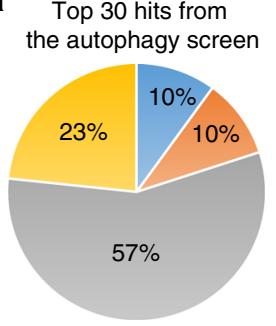

- Drugs

- Synthetic compounds with known functions

- Natural product fraction

= Synthetic compounds with unknown functions
DMSO

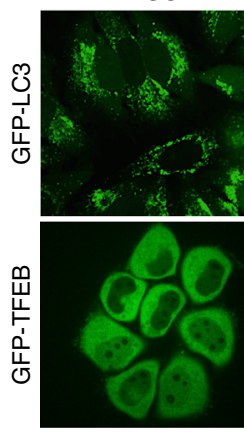

DG

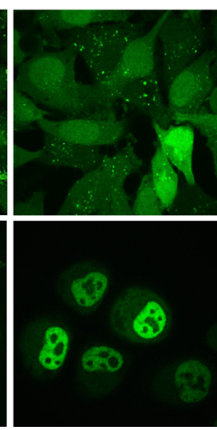

$A D$

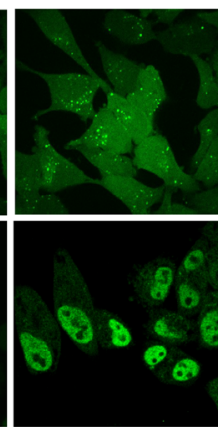

IKA

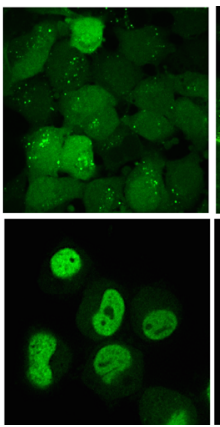

Baf A1

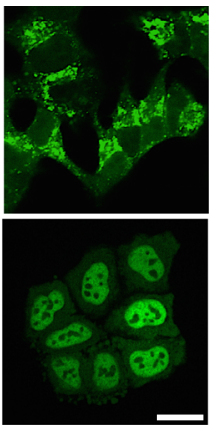

Fig. 2 High-throughput screen for small-molecule agonists of TFEB. a Pie charts showing the composition of the top 30 hits from the autophagy screen (left) and the top 18 hits from the TFEB screen (right). The top 18 hits include 3 FDA-approved drugs (digoxin, proscillaridin A, and digoxingenin), 11 natural product fractions and 4 synthetic small molecules (including alexidine dihydrochloride and cycloheximide). b Robust $Z$-score plot of the top 18 chemicals in the TFEB screen that overlap with the top 30 hits from the autophagy screen. c Representative images of GFP-LC3 and GFP-TFEB HeLa cells treated with $370 \mathrm{nM}$ DG, $3.3 \mu \mathrm{M} \mathrm{AD}$, and IKA and $50 \mathrm{nM}$ bafilomycin A1 (Baf A1). GFP-LC3 HeLa cells were pretreated with UPS 4.4 prior to a 4 h compound exposure. Baf A1, which blocks autolysosomal degradation through inhibition of vacuolar ATPases, was used as a negative control. In GFP-TFEB HeLa cells, the same concentration of compounds was used without $\mathrm{UPS}_{4.4}$, while Baf A1 was used as a positive control. Scale bars, $20 \mu \mathrm{m}$ 
a

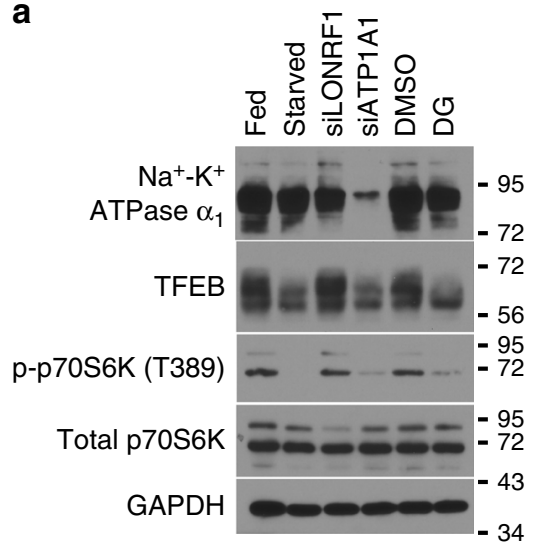

b

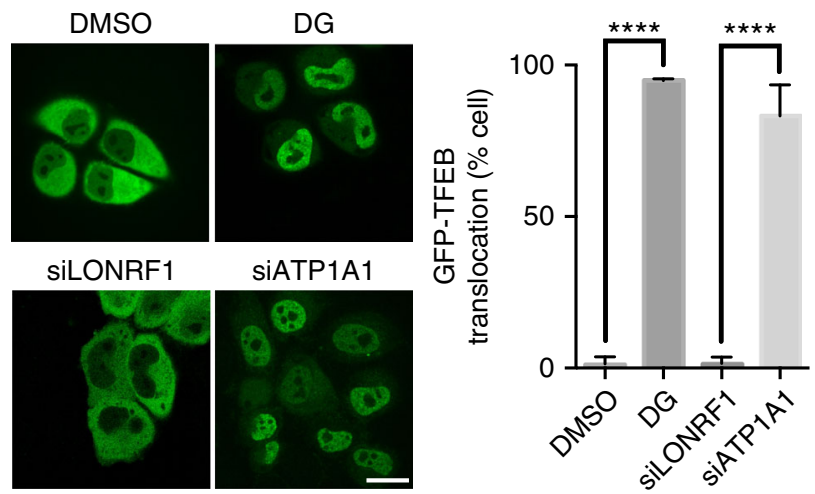

d

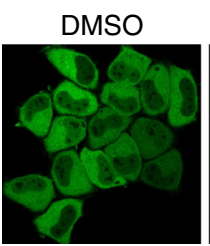

SiLONRF1
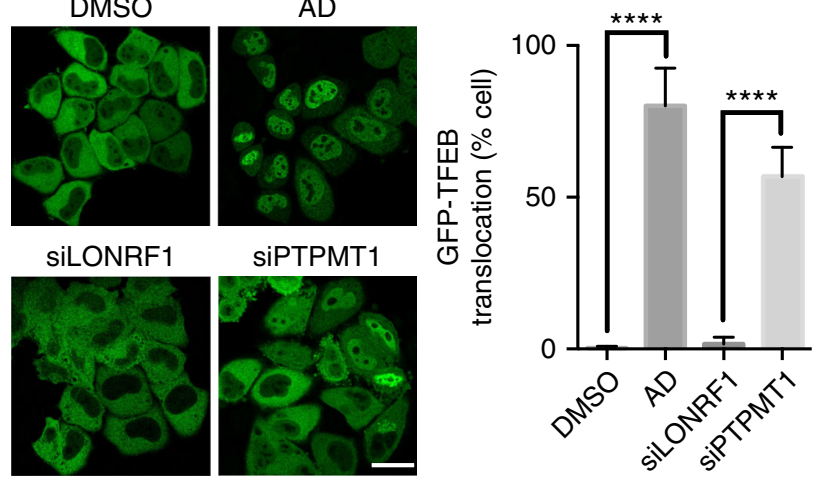

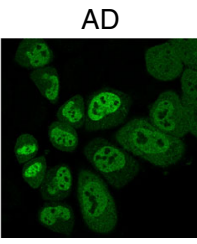

SiPTPMT1

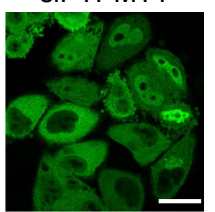

e

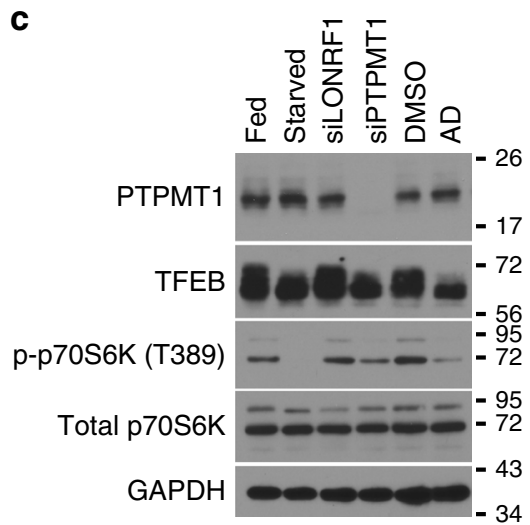

DG
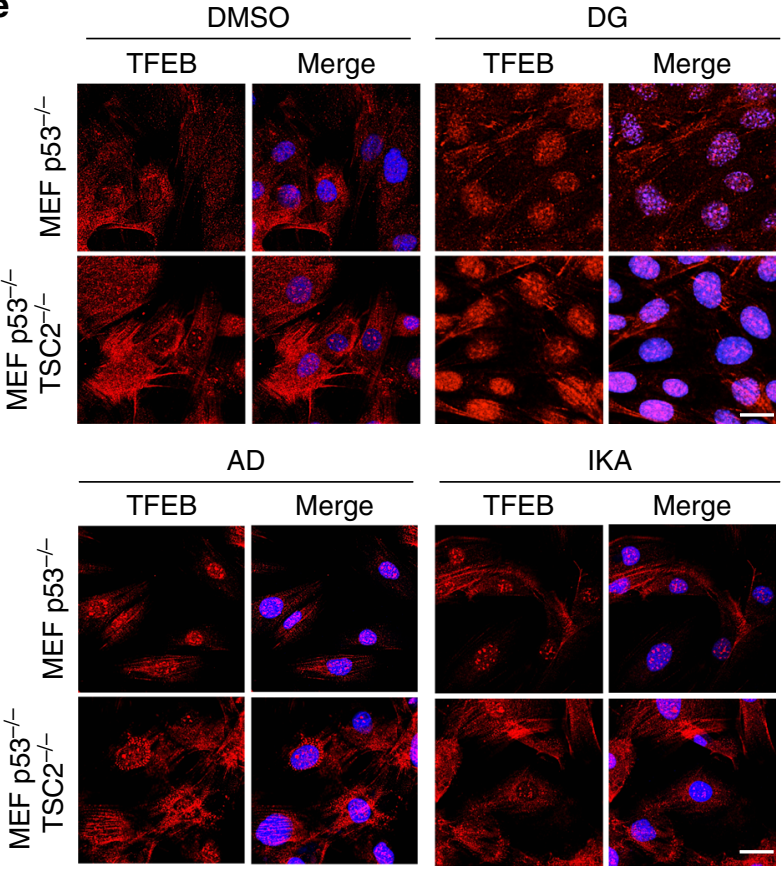

KA
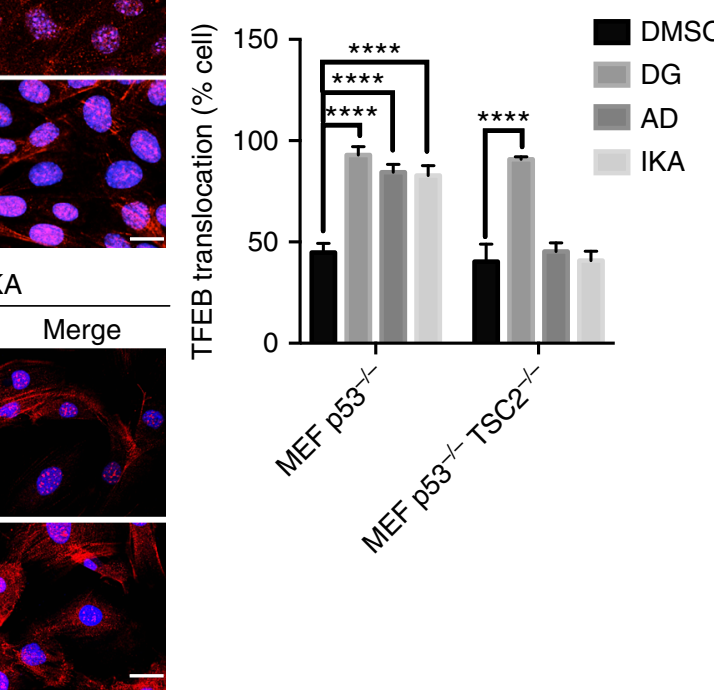

Fig. 3 Target-dependent activation of TFEB and inhibition of mTORC1 by DG, AD, and IKA. a-d siRNA-mediated depletion of the $\alpha_{1}$ subunit of Na ${ }^{+}-K$ ${ }^{+}$-ATPase (a) and PTPMT1 (c) mimics the molecular weight shift of TFEB and inhibition of mTORC1 as seen in the immunoblots of DG-treated (a) and ADtreated (c) and nutrient-deprived cells (positive controls). Representative confocal images of GFP-TFEB HeLa cells treated with DG, siATP1A1 (b) AD, siPTPMT1 (d) and their corresponding controls. siRNA against LON peptidase N-terminal domain and ring finger 1 (LONRF1) was used as a negative control siRNA. The graphs represent the percentage of cells with GFP-TFEB translocation under these conditions (mean \pm s.d. for $n=3$ independent experiments, ${ }^{\star \star \star \star} p<0.0001$ by two-way ANOVA). Scale bar, $20 \mu \mathrm{m}$. e To test if the compound-mediated inhibition of mTORC1 was dependent on the well-known negative regulator TSC2, we employed $p 53^{-/-}$and $p 53^{-/-}, \mathrm{TSC}^{-/-}$mouse embryonic fibroblasts (MEFs). Endogenous TFEB in cells treated with 370.4 $\mathrm{nM}$ DG, $3.3 \mu \mathrm{M} \mathrm{AD}$, or IKA or DMSO was examined by immunofluorescent staining. TFEB translocation percentage was quantified in the bar graph (mean \pm s.d. for $n=3$ independent experiments, ${ }^{\star \star \star \star} p<0.0001$ by two-way ANOVA). Scale bars, $20 \mu \mathrm{m}$ 
a
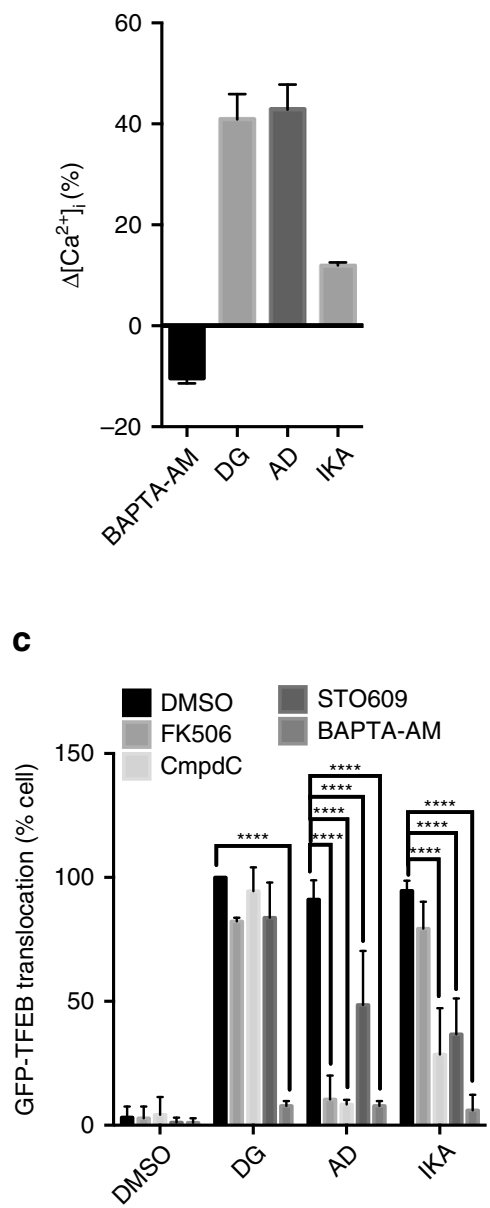

b

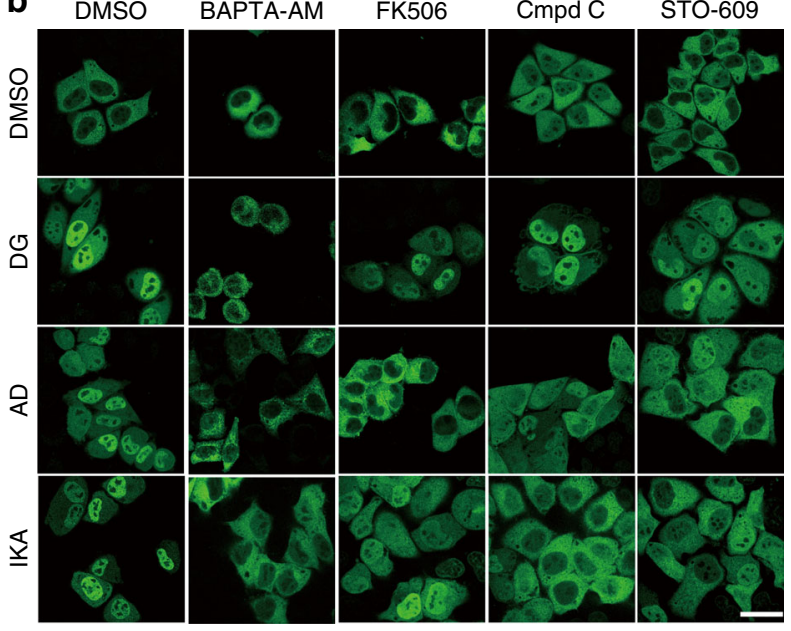

d SILONRF' siPPP3CB

e

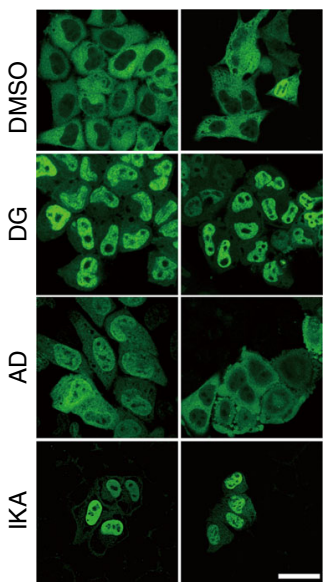

f

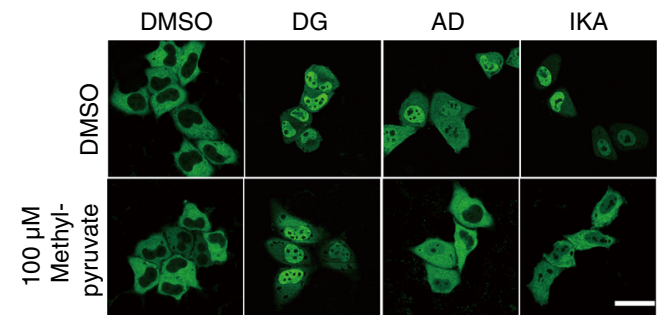

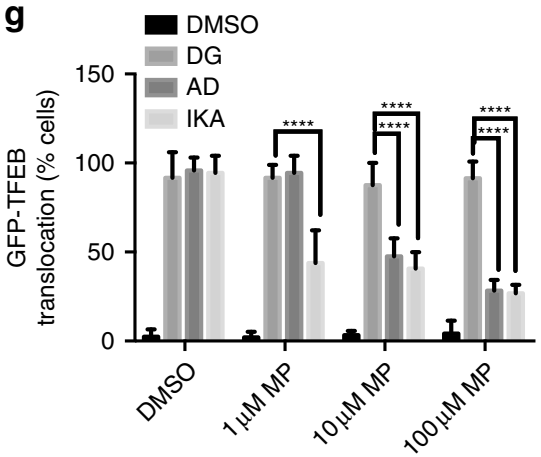

Fig. 4 Engagement of distinct $\mathrm{Ca}^{2+}$ pathways by small-molecule agonists of TFEB. a Intracellular $\mathrm{Ca}^{2+}$ concentration was measured in wild-type HeLa cells treated with $5 \mu \mathrm{M}$ BAPTA-AM, $370 \mathrm{nM}$ DG, $3.3 \mu \mathrm{M}$ AD, and IKA using Fura-2-AM, and the concentration difference between compound-treated and DMSO-treated cells was normalized to the $\mathrm{Ca}^{2+}$ concentration in DMSO-treated cells ( $n=3$ independent experiments). $\mathbf{b}$ Confocal images of GFP-TFEB

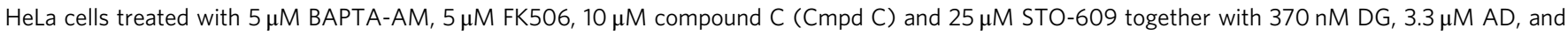
IKA for $4 \mathrm{~h}$. Scale bar, $20 \mu \mathrm{m}$. c The graph represents the percentage of cells with GFP-TFEB translocation in $\mathbf{b}$ (mean \pm s.d. for $n=3$ independent experiments, ${ }^{\star \star \star \star} p<0.0001$ by two-way ANOVA). d Representative images of GFP-TFEB HeLa cells treated with DG, AD, and IKA together with control siRNA (siLONRF1) treatment, siRNA-mediated inhibition of PPP3CB (siPPP3CB) or in combination with $5 \mu \mathrm{M}$ calcineurin inhibitor FK506 for $4 \mathrm{~h}$. Scale bar, $20 \mu \mathrm{m}$. e The graph represents the percentage of cells with GFP-TFEB translocation in $\mathbf{d}$ (mean \pm s.d. for $n=3$ independent experiments, ${ }^{\star \star \star \star} p<0.0001$ by two-way ANOVA). Inset shows the immunoblot of HeLa cells treated with siLONRF1 or siPPP3CB. $\mathbf{f}$ Cell-permeable pyruvate can reverse the TFEB nuclear translocation induced by AD and IKA, but not DG. Scale bar, $20 \mu \mathrm{m}$. $\mathbf{g}$ The graph represents the percentage of cells with GFP-TFEB translocation in $\mathbf{f}$ (mean \pm s.d. for $n=3$ independent experiments, ${ }^{\star \star \star \star} p<0.0001$ by two-way ANOVA) 
which promotes robust induction of autophagosome maturation in response to nutrient starvation, and is amenable to chemical interrogation. We, therefore, leveraged this readout to evaluate 15,000 chemical entities for cellular activity that mimics nutrient starvation (Supplementary Fig. 2a-d). Thirty (out of 80) primary hits were confirmed by independent analyses (Fig. 2a and Supplementary Fig. 2b, e). We next evaluated these compounds for effects on TFEB activity under nutrient replete culture conditions. Transcriptional competence of TFEB is modulated by physical compartmentalization in the cytoplasm (off-state) versus the nucleus (on-state). Chemically induced TFEB nuclear translocation was, therefore, monitored using the fluorescent intensity ratio of nuclear versus cytoplasmic GFP-TFEB (Supplementary Fig. $2 \mathrm{f}-\mathrm{h}$ ). The quantitative robustness of these assays was indicated by a low coefficient of variance (\% CV) and a high Z-factor calculated for the neutral control condition (Supplementary Fig. 2i). The top scoring hits included three cardiac glycosides (digoxin, proscillaridin A, and digoxigenin), two natural-product fractions (SW201073 and SW199954), and the synthetic smallmolecule alexidine dihydrochloride (Fig. 2a, b and Supplementary Fig. 2j). Structure determination revealed the bioactive component of SW201073 is identical to ikarugamycin (Supplementary Fig. 2k, 1), a macrocyclic antibiotic first isolated from Streptomyces phaeochromogenes ${ }^{25}$. We chose to further pursue mechanism of action studies with digoxin (DG), alexidine dihydrochloride (AD), and ikarugamycin (IKA), as they were the top-ranking representatives from three distinct categories of chemical compounds.

Consistent with the primary screen results, all three compounds promoted autophagic flux and activated TFEB in a dosedependent manner as indicated by clearance of $\mathrm{UPS}_{4.4}$-dependent accumulation of GFP-LC3 puncta; increased autophagic flux (conversion of LC3-I to LC3-II); turnover of the long-lived autophagy adaptor protein p62/SQSTM $1^{26}$; and translocation of GFP-TFEB from cytosol to nucleus (Fig. 2c and Supplementary Fig. $2 \mathrm{~m}-\mathrm{r}$ ). The activity of DG was consistent with isolation of this compound as a hit in a Prestwick-library-focused screen for compounds that promote bulk autophagy ${ }^{27}$. The EC50 of these compounds for promotion of p62/SQSTM1 clearance was generally higher than corresponding TFEB nuclear accumulation EC50s consistent with time and signal delta between TFEB activation and transcription-dependent autophagy induction (Supplementary Fig. 2m, p, q). Activation of TFEB is known to induce the expression of numerous lysosomal and autophagic genes to promote lysosomal biogenesis and maturation ${ }^{9}, 11$. Indeed, all three compounds induced the expression of TFEB target genes ${ }^{7}$ (Supplementary Fig. 2s), and accelerated cellular endosomal maturation rates as indicated by accumulation of acidified organelles (Supplementary Fig. 2t) and activation of cathepsin B (Supplementary Fig. 2u). Furthermore, shortinterfering RNA (siRNA)-mediated TFEB depletion was sufficient to inhibit the capacity of the compounds to induce autophagic flux or activate TFEB target genes (Supplementary Fig. $2 \mathrm{v}, \mathrm{x}$ ). Depletion of both TFEB and its homolog, TFE3, further hindered cellular responses to all three compounds (Supplementary Fig. 2 $\mathrm{w}, \mathrm{x})$.

Distinct engagement of mTORC1 by TFEB agonists. Known direct molecular targets of DG and AD in cells are the $\mathrm{Na}^{+}-\mathrm{K}^{+}$ ATPase $\alpha_{1}$ subunit (encoded by ATP1A1) ${ }^{28}$ and the protein tyrosine phosphatase mitochondrial 1 (PTPMT1) ${ }^{29}$, respectively. siRNA-mediated depletion of these targets recapitulated GFP-TFEB nuclear translocation in nutrient replete culture conditions, suggesting DG (Fig. 3a, b) and AD (Fig. 3c, d) engage TFEB through their reported cellular targets. The activity of
TFEB is known to be directly regulated by the kinase mTORC1 and the phosphatase calcineurin, where mTORC1 directly phosphorylates TFEB S142 and S211 to promote cytosolic sequestration via phospho-serine-dependent interaction with 143-3 proteins $^{11,13,17}$ while calcineurin dephosphorylates TFEB and promotes its nuclear localization. To begin to parse how these targets engage TFEB, we first examined mTOR pathway activity. Exposure to all three compounds as well as depletion of the known compound targets, $\mathrm{Na}^{+}-\mathrm{K}^{+}$ATPase $\alpha_{1}$ subunit or PTPMT1, resulted in detectable dephosphorylation of the mTORC1 substrate p70 S6 kinase (p70S6K) under nutrient replete culture conditions, consistent with an inhibition of mTORC1 activity that would normally occur in response to nutrient starvation (Fig. 3a, c). However, inhibition of mTORC1 and activation of TFEB by DG and proscillaridin A (PA, one of the cardiac glycoside hits) was independent of the nutrientresponsive mTOR inhibitory component tuberous sclerosis 2 (TSC2) (Fig. 3e and Supplementary Fig. 3a-c). By contrast, the impact of AD and IKA on mTORC1 pathway activity was TSC2dependent (Fig. 3e and Supplementary Fig 3d). This suggested distinct mechanisms of engagement of mTORC1 and TFEB by $\mathrm{DG}, \mathrm{AD}$, and IKA.

Disparate $\mathrm{Ca}^{2+}$-dependent mechanisms mediate AD, DG, and IKA induction of TFEB. A key TFEB activation mechanism is calcium/calmodulin-dependent dephosphorylation of TFEB (S142) by the calcineurin protein phosphatase ${ }^{12}$. DG, AD, or IKA exposure at $\sim \mathrm{TFEB}_{\mathrm{EC} 90}$ was sufficient to induce accumulation of cytosolic calcium as indicated by the quantification of Fura-2 imaging (Fig. 4a), and $\mathrm{Ca}^{2+}$ chelation by BAPTA-AM was sufficient to block TFEB activation and reverse mTORC1 inhibition by all three compounds (Fig. $4 \mathrm{~b}$ and Supplementary Fig. 4a). Direct inhibition of calcineurin with FK506 ${ }^{30,31}$, cyclosporine A $(\mathrm{CsA})^{30,31}$, or RNAi-mediated depletion of the calcineurin catalytic subunit (PPP3CB) was also sufficient to block TFEB activation by $A D$, suggesting small-molecule inhibition of PTPMT1 activates TFEB via mobilization of calcineurin catalytic activity (Fig. 4b-e and Supplementary Fig. 4b, c). DG-induced TFEB activation was resistant to calcineurin perturbation at EC90, but was inhibited to some extent at EC50 (Fig. 4b-e and Supplementary Fig. 4c). In contrast, IKA-induced TFEB activation was calcineurin independent (Fig. 4b-e and Supplementary Fig 4c). Consistent with that, $\mathrm{AD}$, but not DG and IKA, induced dosedependent nuclear translocation of nuclear factor of activated $\mathrm{T}$ cells (NFAT) ${ }^{32}$, which is known to depend on the activity of calcineurin (Supplementary Fig. 4d).

An additional primary response to both elevated cytosolic $\mathrm{Ca}^{2+}$ and nutrient starvation is activation of AMP-activated protein kinase $(\mathrm{AMPK})^{33}$. AMPK mediates biological responses to caloric restriction through both mTOR-dependent and mTORindependent mechanisms and is engaged directly by calcium/ calmodulin-dependent protein kinase kinase beta (CaMKK $\beta$ ) upon elevation of cytosolic $\mathrm{Ca}^{2+}$. As expected, chemical activation of AMPK either directly with 5-aminoimidazole-4-carboxamide ribonucleotide (AICAR) ${ }^{34}$ or indirectly with metformin ${ }^{35}$ was sufficient to induce TFEB nuclear accumulation (Supplementary Fig. 4e). Dorsomorphin (Compound C), an AMPK inhibitor and STO-609, a CaMKK $\beta$ inhibitor ${ }^{36}$, both significantly inhibited TFEB nuclear translocation induced by IKA and AD, but not DG. Furthermore, IKA and $\mathrm{AD}$, but not $\mathrm{DG}$, induced activating phosphorylation of AMPK and its downstream substrate acetylCoA carboxylase (ACC) in a dose-dependent manner (Supplementary Fig. 4f, g). Finally, the effects of $\mathrm{AD}$ and IKA, but not DG, on TFEB can be reversed by addition of cell-permeable pyruvate, which increases intracellular ATP level and promotes 
a

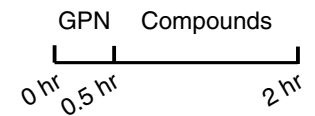

GPN:
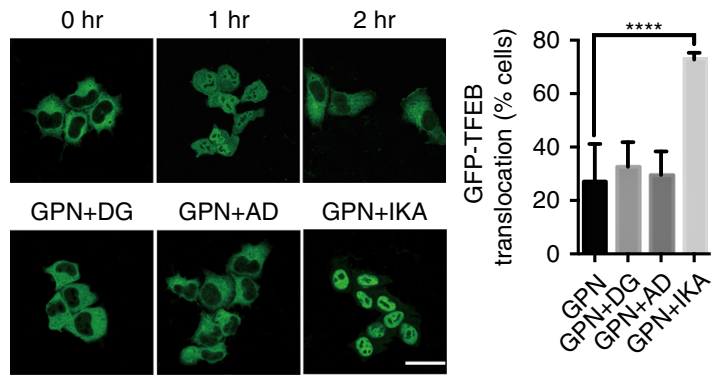

b

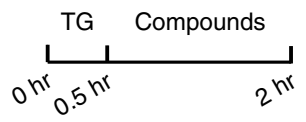

TG:
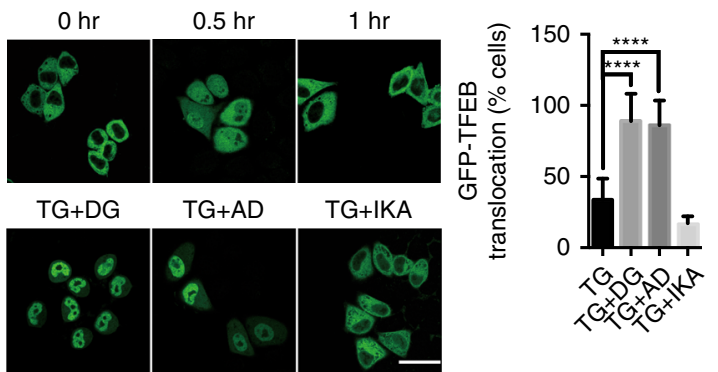

C
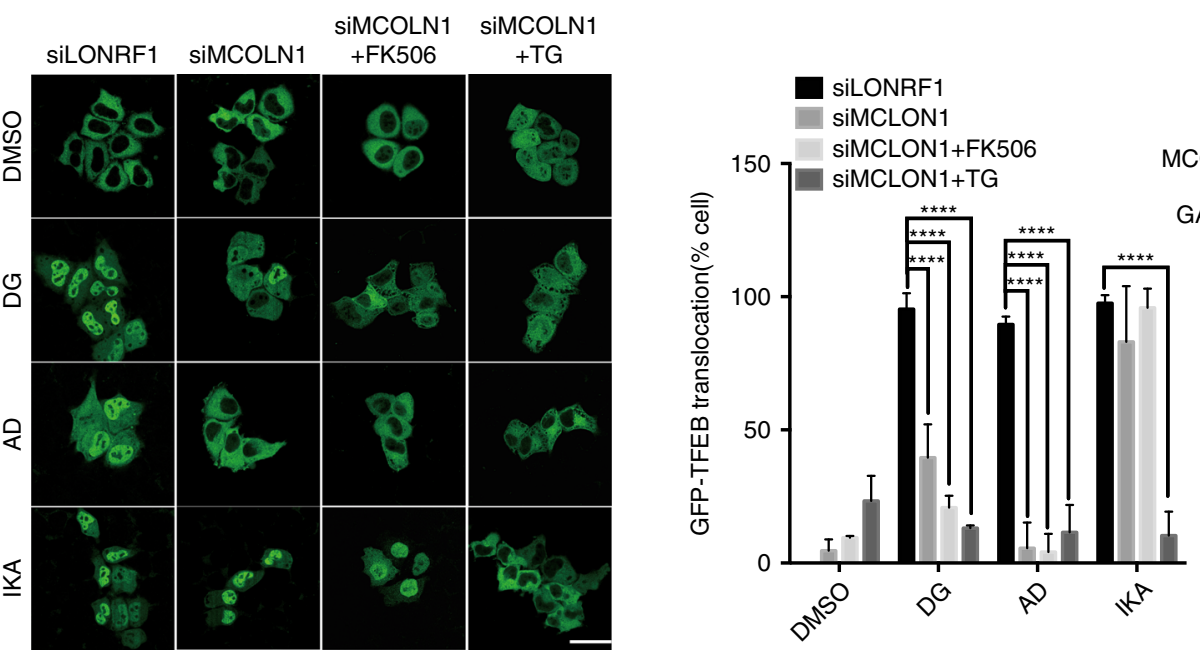

d

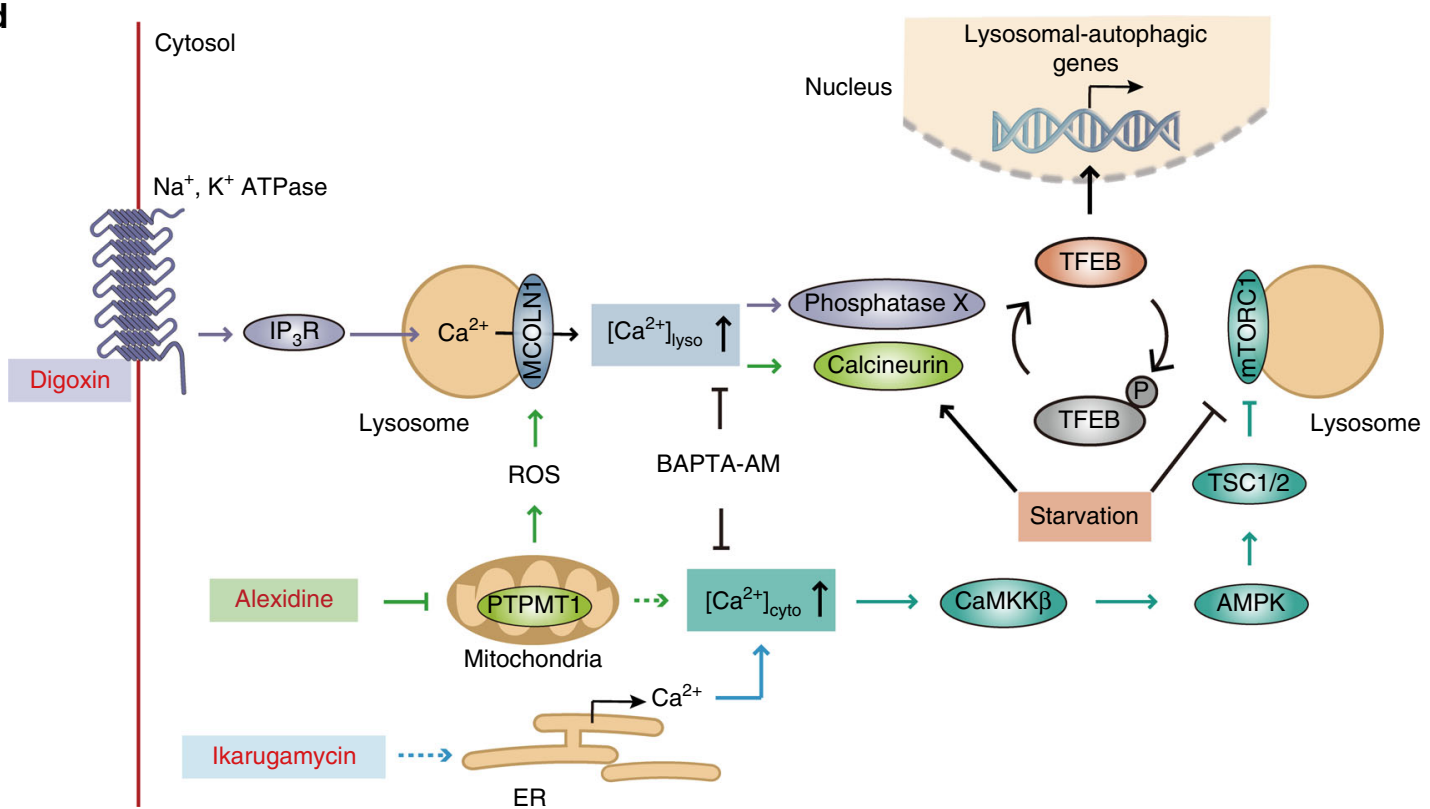

inactivation of AMPK (Fig. 4f, g). These observations indicate that distinct $\mathrm{Ca}^{2+}$-dependent mechanisms mediate $\mathrm{AD}, \mathrm{DG}$, and IKA induction of TFEB. AD activity is calcinuerin- and AMPKdependent, IKA is calcinuerin-independent but AMPK-dependent, and DG is relatively independent of both calcinuerin and AMPK.
Different $\mathrm{Ca}^{2+}$ stores contribute to TFEB activation by DG and AD versus IKA. Lysosomes, mitochondria and endoplasmic reticuli (ER) are the major compartmentalized $\mathrm{Ca}^{2+}$ stores in cells ${ }^{37}$. The seemingly distinct mechanistic links between chemically induced cytosolic $\mathrm{Ca}^{2+}$ and TFEB activation lead us to consider discrete $\mathrm{Ca}^{2+}$ sources as a potential specificity 
determinant. Glycyl-L-phenylalanine 2-napthylamide (GPN) is a lysosome-disrupting agent that is commonly used to deplete lysosome-specific $\mathrm{Ca}^{2+}$ stores $^{38}$. A 30 min pretreatment of GPN was sufficient to disrupt TFEB nuclear translocation induced by $\mathrm{DG}$ and $\mathrm{AD}$, but had almost no effect on IKA-treated cells (Fig. 5a). In contrast, ER-specific depletion of $\mathrm{Ca}^{2+}$ by acute treatment with thapsigargin (TG), a specific inhibitor of ER $\mathrm{Ca}^{2+}$ ATPase SERCA pump ${ }^{39}$, selectively decreased TFEB nuclear translocation induced by IKA (Fig. 5b). This suggests selective perturbation of lysosomal calcium pools by DG and AD versus ER calcium pools by IKA. Notably, RNAi-mediated depletion of the principal $\mathrm{Ca}^{2+}$ channel in lysosomes, mucolipin-1 (MCOLN1), suppressed TFEB nuclear translocation induced by DG and AD, but not IKA (Fig. 5c). MCOLN1 can be activated by ROS in the cells and thus activate TFEB in a lysosomal $\mathrm{Ca}^{2}$ ${ }^{+} /$calcineurin-dependent manner ${ }^{40}$. As AD impairs mitochondrial function through targeting PTPMT1, we tested intracellular ROS levels as a potential explanation for the consequences of $\mathrm{AD}$ on TFEB activation. Consistent with this hypothesis, tert-Butyl hydroperoxide (TBHP) and AD induced comparable ROS production and TFEB nuclear translocation. Importantly, the effects of both compounds on these phenotypes were abolished by the membrane-permeable antioxidant n-acetyl-cysteine (NAC) (Supplementary Fig. 5a-c). With respect to DG, we noted that cardiac glycosides have been reported to promote the binding of $\mathrm{Na}^{+}-\mathrm{K}^{+}$ATPase to IP3R, which can in turn induce downstream $\mathrm{Ca}^{2+}$ release through IP3R ${ }^{41}$ and refuel lysosomal $\mathrm{Ca}^{2+}$ store. A relatively specific IP3R inhibitor Xestosporin C (Xesto), as well as a siRNA-mediated depletion of type 1 IP3R (IP3R1), profoundly attenuated TFEB nuclear translocation induced by IKA, AD, and DG in a time-dependent manner (Supplementary Fig. 5d-i) consistent with the direct engagement of ER- versus lysosomespecific (indirectly through ER) $\mathrm{Ca}^{2+}$ by these compounds. Taken together, these observations indicate that distinct calcium stores mediate TFEB activation by $\mathrm{DG}$ and $\mathrm{AD}$ versus IKA (Fig. 5d).

TFEB agonists mitigate metabolic syndromes and extend lifespan in vivo. In animals, TFEB plays a key role in promoting lipid metabolism during starvation, at least in part through global transcriptional activation of peroxisome proliferator-activated receptor $\gamma$ coactivator $1 \quad \alpha \quad(P \operatorname{pargcl} \alpha)$ and peroxisome proliferator-activated receptor $\alpha(\operatorname{Ppar} 1 \alpha)^{7,42}$. Consistent with physiologically pertinent TFEB activation, DG, AD, and IKA significantly ameliorated oleic acid-induced lipid accumulation in human hepatocytes (Fig. 6a). Importantly, oral administration of DG normalized body weight, body composition and circulating cholesterol, triglycerides, glucose and insulin levels in mice challenged with a high-fat diet (Fig. 6b-g). In contrast, DG did not alter the body weight of the lean mice (Supplementary Fig. 6a). For in vivo analysis of $\mathrm{AD}$ and IKA, compounds were encapsulated into biocompatible, biodegradable polyethylene glycol- $b$-poly (D, L-lactic acid) (PEG-PLA) nanoparticles that are liver-trophic ${ }^{43,44}$ (Supplementary Table 1). Controlled and sustained release of AD and IKA persisted for more than 2 days in an in vitro setting that mimics the in vivo environment (Supplementary Fig. 6b), supporting the 3-times-a-week treatment regimen. Like DG, significant normalization of body weight/ composition and blood chemistry was observed upon high-fat challenge relative to control groups (Fig. 6c-g and Supplementary Fig. $6 \mathrm{c}, \mathrm{d}$ ). Moreover, compound-treated mice also displayed improved glucose and insulin tolerance relative to control animals (Fig. 6h). Liver histology revealed amelioration of high-fat dietinduced steatosis, which corresponded to upregulation of Ppargc1 $\alpha$, Pparl $\alpha$, and Fgf2 $1^{45-47}$ by DG, AD and IKA (Fig. 6i and Supplementary Fig. 6e). Compound treatment also reversed p62/SQSTM1 accumulation in hepatocytes, suggesting enhanced autophagic flux in these mice (Fig. 6i). No obvious toxicity to major organs was observed in any treatment group, nor was it observed in the in vitro experiments (Supplementary Fig. 6f, g). An overnight fast in mice is sufficient to induce a transient increase in hepatic lipid accumulation as a consequence of adipose tissue lipolysis ${ }^{48}$, a phenotype that is exacerbated by chloroquine (CQ) (Supplementary Fig. 6h, i). Notably, this effect can be improved by co-administration of DG, consistent with an enhanced endolysosomal function engaged by TFEB in hepatocytes (Supplementary Fig. 6j-l); an effect also consistent with DG-dependent reduction in p62/SQSTM1 accumulation (Supplementary Fig. 6h). These observations indicate that TFEB activation induced by $\mathrm{DG}, \mathrm{AD}$, and IKA engages lipid catabolism and can revert physiologically pertinent metabolic syndromes.

Importantly, TFEB has recently been shown to be required for the lifespan extension induced by starvation/calorie restriction and autophagy in vivo ${ }^{8}$. Thus, we investigated if chemically activated TFEB was sufficient to modulate lifespan. The nematode C. elegans was selected as a relevant animal model, as the $C$. elegans TFEB ortholog HLH-30 engages the CLEAR motif to induce the expression of orthologous TFEB targets and autophagy in vivo ${ }^{7,8}$. A sterile fem-1 (hc17ts) and dal-1 (dt2300) background was chosen to facilitate the lifespan assay and accumulation of xenobiotics ${ }^{49}$, respectively. fem-1(-) animals have temperaturesensitive fertility defects, which simplifies lifespan analysis as mothers do not need to be continuously separated from progeny. dal-1(-) worms are healthy but have permissive oral chemical bioavailability via enhanced intestinal absorption. Among the

\footnotetext{
Fig. 5 Distinct $\mathrm{Ca}^{2+}$-dependence of small-molecular agonists of TFEB. a, b GFP-TFEB HeLa cells before and after a 30-min treatment of 200 $\mu$ M GPN (upper panel of $\mathbf{a}$ ) or $300 \mathrm{nM}$ TG (upper panel of $\mathbf{b}$ ) and cells pretreated 30 min with GPN followed by a treatment with DG, AD, and IKA (lower panel). The graph represents the percentage of cells with GFP-TFEB translocation under these conditions (mean \pm s.d. for $n=3$ independent experiments, ${ }^{\star \star \star \star} p<0.0001$ by one-way ANOVA). Scale bar, $20 \mu \mathrm{m}$. c Representative images of GFP-TFEB HeLa cells treated with DG, AD, and IKA together with control siRNA (siLONRF1) treatment, siRNA-mediated inhibition of MCOLN1 (siMCOLN1) or in combination with $5 \mu \mathrm{M}$ FK506 (4h) and $300 \mathrm{nM}$ TG (30 min pretreatment). The graph represents the percentage of cells with GFP-TFEB translocation under these conditions (mean $\pm \mathrm{s}$.d. for $n=3$ independent experiments, ${ }^{\star \star \star \star} p<0.0001$ by two-way ANOVA). Scale bar, $20 \mu \mathrm{m}$. Inset shows the immunoblot of cells treated with siLONRF1 or siMCOLN1.

d Schematics of proposed mechanism of actions of $D G, A D$, and IKA. Cardiac glycosides, such as DG, promote binding of their molecular target ( $\mathrm{Na}^{+}-\mathrm{K}$ ${ }^{+}$-ATPase) to IP3R. IP3R-dependent ER $\mathrm{Ca}^{2+}$ release then recharges lysosomal $\mathrm{Ca}^{2+}$ stores through an unclear mechanism, enabling lysosomal $\mathrm{Ca}^{2+}$ release through mucolipin-1 (MCOLN1). AD targets PTPMT1 in mitochondria to perturb mitochondrial function and induce ROS release. The lysosomal Ca ${ }^{2+}$ channel mucolipin-1 responds to elevated ROS, which results in a lysosomal $\mathrm{Ca}^{2+}$ release. This activates calcineurin and likely additional unknown phosphatases, which de-phosphorylate TFEB and promote nuclear translocation. Furthermore, mTORC1 maintains inhibitory TFEB phosphorylation under nutrient-rich conditions. AD and IKA both increase cytosolic $\mathrm{Ca}^{2+}$ levels resulting in CaMKK $\beta$ and AMPK pathway activation, which in turn negatively regulates $\mathrm{mTORC1}$ to promote TFEB activation. DG also inhibits the activity of $\mathrm{mTORC1}$ through an unknown mechanism. Activation of TFEB promotes lysosomal biogenesis and autophagy and upregulates genes promoting lipid metabolism. DG-, AD-, and IKA-related proteins/pathways were coded in purple, green, and light blue. Shared proteins/pathways between DG and AD or AD and IKA were coded in cyan and dark blue
} 
a
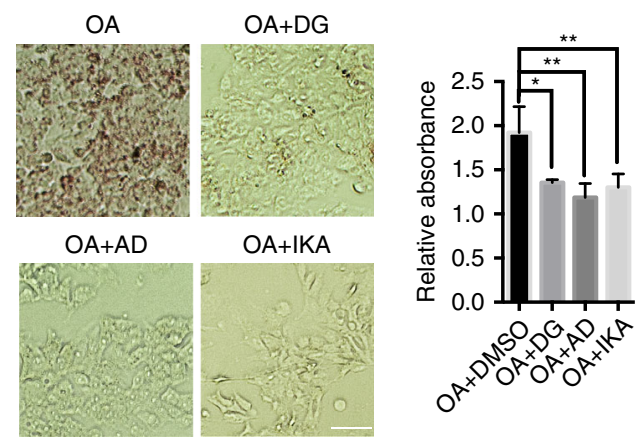

b

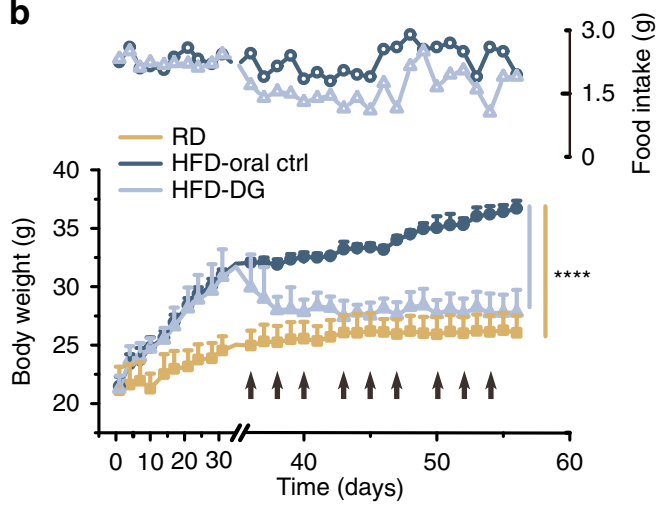

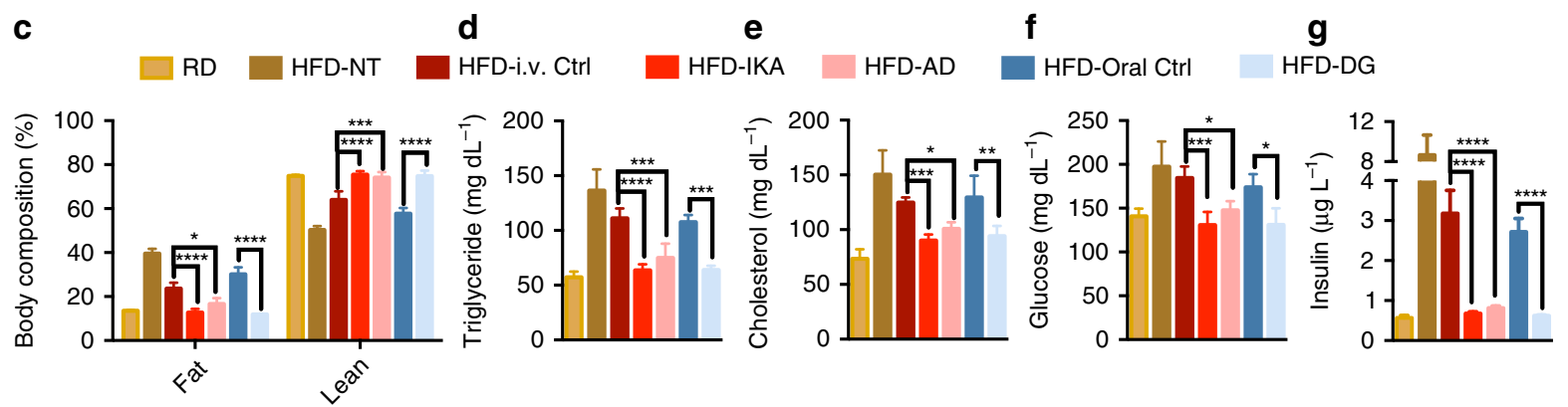
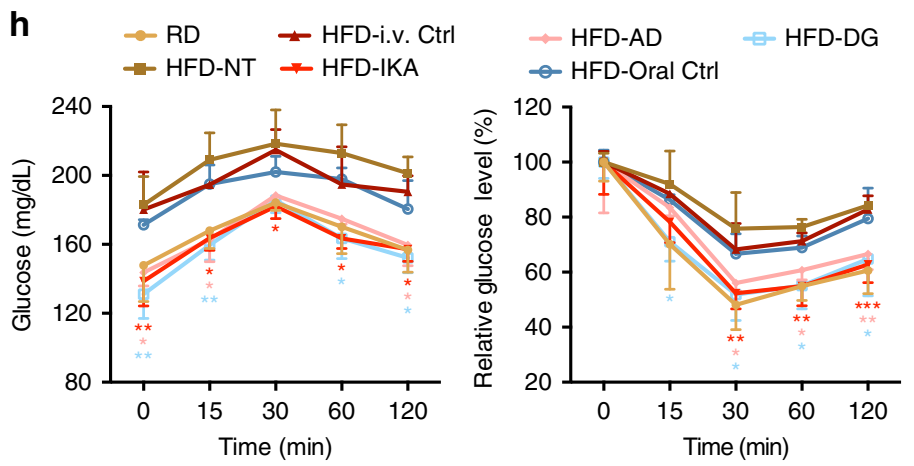

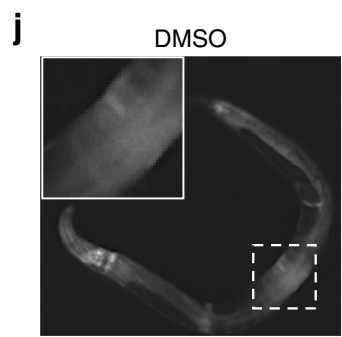

IKA

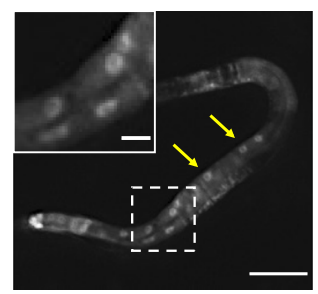

\section{$\mathbf{k}$}

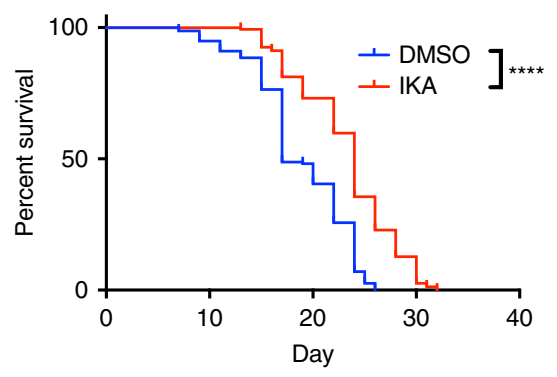

I Liver H\&E Liver ORO Liver p62 IHC

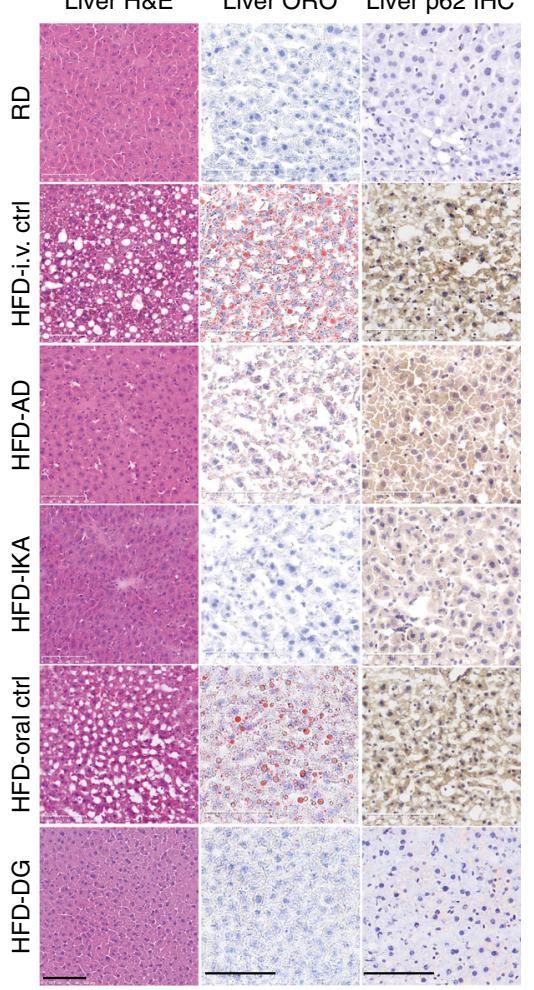

three compounds, IKA-treatment was found to induce nuclear accumulation of GFP-tagged HLH30 within intestinal cells in adults (Fig. 6j). Notably, IKA significantly extended the lifespan of the fem-1(-);dal-1(-) animals (Fig. 6k and Supplementary Table 2).

\section{Discussion}

In this study, we leveraged a facile high-throughput phenotypic screening strategy to identify small-molecule TFEB agonists. Mechanism of action analyses of the lead hits has revealed distinct mechanistic mobilization of intracellular calcium stores that 
can result in productive engagement of TFEB activity in cells. These mechanisms presumably translate, at least in part, to in vivo activity corresponding to amelioration of diet-induced lipid metabolic disorders and lifespan extension.

The conventional target-centric approach for drug discovery, without consideration of optimal cellular mechanisms of action, may result in the current high attrition rates and low productivity in pharmaceutical research and development. Herein, we carefully designed a phenotypic screen for TFEB agonists with therapeutic potential. To avoid lysosomal stressors such as bafilomycin A1 and chloroquine, we first selected for agents that promote autophagic flux in a GFP-labeled LC3 cell model. A broad dynamic activity window was achieved by using UPS nanobuffers that clamp the luminal $\mathrm{pH}$ of endocytic organelles and thus arrest them at distinct maturation stages (Fig. 1a). Compared to membrane-permeable small-molecular bases (e.g., chloroquine and $\mathrm{NH}_{4} \mathrm{Cl}$, Fig. 1b), the UPS collection facilitates high-resolution characterization of luminal $\mathrm{pH}$-specific cell signaling and metabolic processes ${ }^{23}$. UPS 4.4 was leveraged for these studies because it selectively perturbs lysosomal degradation of macromolecules and maximally preserves signaling functions. Moreover, the $\mathrm{UPS}_{4.4}$-dependent arrest of lysosomal $\mathrm{pH}$ can be overcome by enhanced lysosomal proton import via v-ATPase activity. The significantly increased signal window by $\mathrm{UPS}_{4.4}$ allowed improved accuracy and high-throughput screening of a large collection of compounds. A secondary high-content screen for agents that induce TFEB nuclear translocation allowed the discovery of novel TFEB agonists including several FDAapproved drugs, marine-derived natural product fractions and synthetic small molecules.

Starvation-induced autophagy has been shown to be regulated by lysosomal $\mathrm{Ca}^{2+}$-mediated TFEB activation ${ }^{7}$. Here, we find that an orchestrated $\mathrm{Ca}^{2+}$ release from distinct organelles (ER, lysosomes, mitochondria) was selectively induced by each of the three distinct TFEB agonists, which indicates $\mathrm{Ca}^{2+}$ homeostasis as a paramount biological modulator of TFEB activity. These $\mathrm{Ca}^{2+}$ sources selectively engaged either a global $\mathrm{Ca}^{2+}$-sensing CaMKK $\beta$-AMPK pathway or a local $\mathrm{Ca}^{2+}$-sensing MCOLN1calcineurin pathway, to release the "brake" (inhibition of mTORC1 through AMPK) and/or push the "accelerator" (activation of TFEB phosphatases) on the TFEB activation cycle (Fig. 5d). We found that DG-induced lysosomal calcium release through MCOLN1, which is likely refueled by ER calcium release from IP3R. Given the direct contact of cardiac-glycoside-bound $\mathrm{Na}^{+}-\mathrm{K}^{+}$ATPase with the IP3 receptor on $\mathrm{ER}^{50}$, we suspect the mode of action of DG is $\mathrm{Na}^{+}-\mathrm{K}^{+}$ATPase- and IP3R-dependent calcium mobilization and consequent downstream phosphatase activation (Fig. 5d). Surprisingly, DG does not depend on the activity of calcineurin (Fig. $4 \mathrm{~b}-\mathrm{e}$ ), suggesting the presence of an uncharacterized $\mathrm{Ca}^{2+}$-responsive TFEB phosphatase(s). In contrast, we found that the activation of TFEB by AD is through ROS-dependent activation of the MCOLN1-calcineurin pathway via direct perturbation of the mitochondrial protein PTPMT1 (Fig. 4b-e). Whether or not different MCOLN1 activation mechanisms account for engagement of different TFEB phosphatases now presents as an intriguing opportunity for future investigation. Unlike $\mathrm{DG}$ and $\mathrm{AD}$, we found that IKA triggers conventional ER-mediated $\mathrm{Ca}^{2+}$-release to activate the CaMKK $\beta$ AMPK pathway (Fig. $4 \mathrm{~b}, \mathrm{c}$ and Fig. $5 \mathrm{a}-\mathrm{c}$ ). Identification of the direct molecular target of IKA will likely shed new light on mechanisms governing organellar communication and selective mobilization of distinct calcium stores.

TFE3 and TFEB regulate overlapping gene sets and have similar biological effects overexpressed ${ }^{18}$. Furthermore, both are regulated by mTORC1-dependent phosphorylation on lysosomal surfaces and by phosphatase calcineurin ${ }^{10,17-19}$. Given the overlapping mechanistic profiles of TFE3 and TFEB it is likely that the compounds described here can activate both TFEB and TFE3. A recent study examining the physiological roles of TFE3 versus TFEB found partial redundancy in several tissues ${ }^{20}$. However, under conditions such as starvation, high-fat diet and exercise, TFE3 and TFEB are both required and their roles are cooperative. Therefore, the beneficial effects of the compounds we observed in HFD-fed mice are likely a consequence of the combined inhibition of both TFEB and TFE3.

Current lipid-lowering drugs are more targeted toward inhibiting cholesterol synthesis or reabsorption, such as HMG-CoA (3-hydroxy-3-methylglutaryl-coenzyme A) reductase inhibitors ${ }^{51}$, bile-acid binding resins ${ }^{52}$, and cholesterol absorption inhibitors ${ }^{53}$. As the role of lysosomes and autophagy in macromolecule metabolism becomes clearer, boosting this innate cellular clearance machinery, such as the TFEB agonists, offers a new therapeutic strategy for treatment of these diseases. In fact, pharmacological intervention of targets upstream of the TFEBautophagy pathway, such as mTORC1 inhibitors ${ }^{54,55}$ and AMPK activators 56,57 , have already been shown to hold considerable promise to reverse metabolic abnormalities and even to extend lifespan. Genetic studies have already demonstrated the role that TFEB plays under these pathophysiological conditions. The newly discovered small-molecule TFEB agonists will facilitate studies focused on new TFEB biology and will promote viable pharmacological strategies to tackle metabolic syndromes, ageing and age-related diseases.

\section{Methods}

Chemical reagents. The TMR-NHS, Cy5-NHS, BODIPY-NHS, and Cy3.5-NHS esters were purchased from Lumiprobe Corp. (FL, USA). 2-Aminoethyl methacrylate (AMA) was purchased from Polyscience Company. Monomers 2-

\footnotetext{
Fig. 6 Small-molecule agonists of TFEB promote lipid metabolism and extend lifespan in vivo. a Bright-field images showing oil red O (ORO)-stained HepG2 cells treated with $1 \mathrm{mM}$ oleic acid (OA) in combination with $370 \mathrm{nM} \mathrm{DG}, 3.3 \mu \mathrm{M} \mathrm{AD}$, and IKA. The graph was obtained by absorbance reading of ORO using plate reader (bars represent mean \pm s.d. ${ }^{\star} p<0.05,{ }^{\star \star} p<0.01,{ }^{\star \star \star} p<0.001$ by two-way ANOVA). Scale bar, $50 \mu \mathrm{m}$. b Food uptake (open symbols and right $y$-axis) and body weight change (solid symbols and left $y$-axis) of mice fed with regular diet (RD), high-fat diet with oral injection of DG solvent (HFD-oral ctrl) and HFD with DG oral injection (HFD-DG) three times a week starting from Day 35 as indicated by the arrows (bars represent mean \pm s.d. ${ }^{\star} p<0.05,{ }^{\star \star} p<0.01,{ }^{\star \star \star} p<0.001,{ }^{\star \star \star \star} p<0.0001$, HFD-DG compared with HFD-oral ctrl group by using two-way ANOVA). c Whole-body composition analysis (EchoMRI) of the same mice as in $\mathbf{b}$ and Supplementary Fig. $6 \mathbf{c}$, $\mathbf{d}$ after 3 weeks of treatment with compounds or their corresponding controls. $\mathbf{d}$ - $\mathbf{g}$ Total serum triglyceride, cholesterol, glucose, and insulin levels in compound-treated mice or their corresponding control mice after 3 weeks of treatment with compounds or their corresponding controls. $\mathbf{h}$ Glucose levels at indicated time points after glucose challenge (left panels) and insulin challenge (right panels). In b-h, $n=3-5$ mice per group, bars represent mean \pm s.d. ${ }^{*} p<0.05,{ }^{\star \star} p<0.01,{ }^{\star \star \star} p<0.001,{ }^{\star \star \star \star} p<0.0001$ by two-way ANOVA. i Hematoxylin and eosin (H\&E) staining, ORO staining and immunohistochemistry staining against p62 of liver sections isolated from mice after 3 weeks of treatment with or without compounds. HFD-i.v. ctrl, mice injected with empty PEG-PLA nanoparticles. Scale bars, $100 \mu$ m. $\mathbf{j}$ Representative images of HLH-30::GFP nuclear translocation in dal-1(dt2300); sqls19 [hlh-30p::hlh-30::GFP] C. elegans treated with $5 \mu \mathrm{M}$ IKA or DMSO. Insets show enlarged images from the white boxes in the main images. Yellow arrows denote nuclear localized HLH30::GFP. Scale bar, $100 \mu \mathrm{m}$ and $10 \mu \mathrm{m}$ (insets). k The Kaplan-Meier curves of dal-1(2300);fem-1(hc17ts) mutant C. elegans treated with $5 \mu \mathrm{M}$ IKA or DMSO ( $n=2$ independent experiments, ${ }^{\star \star \star \star} p<0.0001$ by log-rank test)
} 
(dibutylamino) ethyl methacrylate (DBA-MA) and 2-(dipentylamino) ethyl methacrylate (D5A-MA) were prepared according to the method described in our previous work, as well as the PEO macroinitiator $\left(\mathrm{MeO}-\mathrm{PEO}_{114}-\mathrm{Br}\right) . \mathrm{N}, \mathrm{N}, \mathrm{N}^{\prime}, \mathrm{N}^{\prime \prime}, \mathrm{N}$ "'-Pentamethyldiethylenetriamine (PMDETA) and poly(ethylene glycol)- $b$-poly(D, L-lactide) (PEG-PLA, $M_{\mathrm{n}} \sim 5,000 \mathrm{Da}$ for each segment) were purchased from Sigma-Aldrich. (2-Hydroxypropyl)- $\beta$-cyclodextrin (HP $\beta C D$ ) was purchased from Fisher Scientific Inc. Amicon ultra-15 centrifugal filter tubes $(\mathrm{MWCO}=100 \mathrm{~K})$ were obtained from Millipore (MA). Other reagents and organic solvents were analytical grade from Sigma-Aldrich or Fisher Scientific Inc. Digoxin, proscillaridin A, alexdine dihydrochloride, ikarugamycin, bafilomycin A1, FK506, cyclosporine A, dorsomorphin (compound C), AICAR, metformin, STO-609, thapsigarigin, $\mathrm{N}$, $\mathrm{N}, \mathrm{N}^{\prime}, \mathrm{N}^{\prime}$-Tetrakis(2-pyridylmethyl)ethylenediamine (TPEN), and oleic acid were purchased from Sigma-Aldrich; Torin 1 was from Tocris Bioscience; Xestosporin C was from Cayman Chemical; BAPTA-AM, Fura-2-AM, calcium calibration buffer kit (with 0 and $10 \mathrm{mM}$ EGTA), Hoechst 33342, CellROX green, tert-Butyl hydroperoxide (TBHP) and NAC were from Invitrogen; Gly-Phe $\beta$-naphthylamide (GPN) from Santa Cruz Biotechnology; Magic Red ${ }^{\mathrm{TM}}-(\mathrm{RR})_{2}$ Cathepsin B assay kit from Marker Gene Technologies, Inc.

Antibodies and immunoblots. The following antibodies were used for immunoblot: GAPDH (cat. 5174, 1:1,000), SQSTM1/p62 (cat. 5114, 1:1,000), TFEB (cat. 4240, 1:1,000), LAMIN A/C (cat. 2023, 1:1000), AMPKo-pThr172 (cat. 2535, 1:1,000), total AMPK $\alpha$ (cat. 5831, 1:1,000), ACC-pS79 (cat. 1:1000), total ACC (cat. 3676, 1:1,000), S6-pS235/236 (cat. 4858, 1:1,000), total S6 (cat. 2217, 1:1,000), $\mathrm{Na}^{+}$, $\mathrm{K}^{+}$-ATPase $\alpha 1$ (cat. 3010, 1:1,000), p70-S6K-pThr389 (cat. 9234, 1:1,000), total p70-S6K (cat. 2708, 1:1,000), TSC2-pThr1462 (cat. 3611, 1:1,000), total TSC2 (cat. 3635, 1:1,000), AKT-pThr308 (cat. 2965, 1:1,000), pan AKT (cat. 4691, 1:1,000), IP3R1 (cat. 3763, 1:1,000), and TFE3 (cat. 14779, 1:1000) were from Cell Signaling Technology and PPP3CB (cat. ab191374, 1:1000) was from Abcam; the following antibody was used for immunofluorescence: TFEB (cat. sc-48784, 1:100) from Santa Cruz Biotechnology and NFAT1 (cat. 5861, 1:100) and LAMP1 (cat. 9091, 1:200) from Cell Signaling Technology; the following antibody was used for immunohistochemistry: p62/SQSTM1 (cat. ab91526, 1:200) from Abcam. Cells were collected in $2 \times$ sample buffer, boiled, and sonicated for immunoblot analysis using standard western blot protocols. Uncropped blots were shown in Supplementary Fig. 7.

Cell culture and siRNA transfection. HeLa cells and MEFs were purchased from ATCC and were cultured in Dulbecco's modified Eagle's medium (DMEM, Invitrogen) with $10 \%$ fetal bovine serum and $1 \%$ antibiotics (Invitrogen). Earle's Balanced Salt Solution (EBSS, 10x, Sigma) was diluted to $1 \mathrm{x}$ with Milli- $\mathrm{Q}$ water supplemented with $2.2 \mathrm{~g} \mathrm{~L}^{-1}$ sodium bicarbonate (Sigma). HeLa cells that stably express GFP-TFEB and GFP-LC3, and $p 53^{-/-}$and $p 53^{-/-}$and $T S C 2^{-/-}$MEFs and HepG2 cells were generous gifts from Dr. Shawn Ferguson (Yale University, USA), Dr. Beth Levine (UT Southwestern Medical Center, USA), Dr. James Brugarolas and Dr. Yihong Wan (UT Southwestern Medical Center, USA), and were cultured under the same conditions as described above. In the GFP-LC3 chemical screen, 2 $\mathrm{mM} \mathrm{NH} 4 \mathrm{Cl}$ (Sigma) was supplemented in DMEM. All cell-based studies were performed with $25 \mathrm{mM}$ HEPES buffer in a humidified chamber with $5 \% \mathrm{CO}_{2}$. All cell lines have been tested for mycoplasma contamination using a MycoFluor ${ }^{\mathrm{TM}}$ Mycoplasma Detection Kit (Invitrogen). RNAi was performed by transfecting siRNA oligos (Dharmacon, Inc.) via reverse transfection using RNAiMax (Life Technologies) according to the manufacturer's instructions. A pool of four siRNA oligos targeting each gene was used to dilute off-target effects. Pools of four siRNAs targeting LONRF1 were used for transfection controls.

Synthesis of PEO-b-PR block copolymers. In a typical procedure using PEO- $b$ $\mathrm{PDBA}_{80}\left(\mathrm{UPS}_{53}\right)$ as an example, DBA-MA $(1.92 \mathrm{~g}, 8 \mathrm{mmol})$, PMDETA $(21 \mu \mathrm{L}, 0.1$ $\mathrm{mmol})$ and $\mathrm{MeO}-\mathrm{PEO}_{114}-\mathrm{Br}(0.5 \mathrm{~g}, 0.1 \mathrm{mmol})$ were charged into a polymerization tube. The monomer and initiator were dissolved in a mixture of 2-propanol $(2 \mathrm{~mL})$ and dimethylformamide $(2 \mathrm{~mL})$. Three cycles of freeze-pump-thaw were performed to remove the oxygen, then $\mathrm{CuBr}(14 \mathrm{mg}, 0.1 \mathrm{mmol})$ was added into the tube protected by nitrogen, and the tube was sealed in vacuo. After $8 \mathrm{~h}$ polymerization at $40{ }^{\circ} \mathrm{C}$, the reaction mixture was diluted in $10 \mathrm{~mL}$ tetrahydrofuran (THF), and the mixture was passed through a neutral $\mathrm{Al}_{2} \mathrm{O}_{3}$ column to remove the catalyst. The organic solvent was removed by rotovap. The residue was dialyzed in distilled water and lyophilized to obtain a white powder.

Preparation of UPS nanoparticle solutions. In a typical procedure, $10 \mathrm{mg}$ UPS polymer was dissolved in $500 \mu \mathrm{L}$ THF $\left(\mathrm{UPS}_{4.4}\right.$ ) or methanol (always-on/OFF-ON $\mathrm{UPS}_{5.3}$ ). For always-on/OFF-ON UPS ${ }_{5.3}$ nanoprobes, BODIPY-conjugated polymer, and Cy3.5-conjugated polymer was mixed with a 3:2 weight ratio. The solution was added to $10 \mathrm{~mL}$ Milli-Q water drop by drop. Four to five filtrations through a micro-ultrafiltration system $(<100 \mathrm{kDa}$, Amicon Ultra filter units, Millipore) were used to remove the organic solvent. The aqueous solution of UPS nanoparticles was sterilized with a $0.22 \mu \mathrm{m}$ filter unit (Millex-GP syringe filter unit, Millipore)
High-throughput GFP-LC3 chemical screen. GFP-LC3 HeLa cells were seeded in 384-well plates. UPS $_{44}$ nanobuffer solutions were added the following day, and compounds $(2.5 \mu \mathrm{M})$ were added for $4 \mathrm{~h}$ on the third day. UPS 4.4 -only cells were used as positive controls and wild-type HeLa cells were used as negative controls. Cells were then fixed with $4 \%$ formaldehyde, stained with $0.01 \%$ Hoechst 33324 , and then sealed and read on with PHERAstar FS HTS microplate reader (BMG LABTECH). A saline-only plate was used to control background signals. Genedata Screener software (GeneData, Inc. Basel, Switzerland) was used to process and analyze the results. For each plate, the raw fluorescence GFP values were normalized with corresponding Hoechst signals after background-correction for all wells. The converted data was then normalized using Equation 1. Normalized well values were then corrected for position artifacts based on GeneData proprietary pattern detection algorithms. Finally, robust $Z$-scores were calculated using Equation 2.

$$
\begin{aligned}
& \text { Normalized data }=\frac{\text { Converted data }_{\text {sample }}-\text { Median of converted dataDMSO }}{\mid \text { Median of converted data } \text { positive control }^{- \text {Median of converted dataDMSO }} \mid} \times 100 \text {. } \\
& \text { Robust } Z \text { score }=\frac{\text { Converted data }_{\text {sample }}-\text { Median of converted data }_{\text {all sample }}}{\text { Converted robust standard deviation }}
\end{aligned}
$$

For the primary screen, each compound was tested as $N=1$, and primary hits were selected with robust $Z$-scores less than -3 . For the validation screen, the primary hits were assayed in triplicate. For each compound, the normalized activity values were condensed to a single value (condensed activity score) using the "Robust Condensing" method in Genedata Screener. The condensed activity is the most representative single value of the triplicates. Thirty compounds with lowest condensed activity values and robust $Z$-score values were selected as the final hits.

High-content GFP-TFEB chemical screen. GFP-TFEB HeLa cells were seeded in 384 -well plates. Compounds were added for $4 \mathrm{~h}$ the following day. Bafilomycin Al $(250 \mathrm{nM})$ treated cells were used as positive controls and dimethyl sulfoxide (DMSO)-treated cells as negative controls. Cells were fixed with $4 \%$ formaldehyde, stained with $0.01 \%$ Hoechst 33324, and then imaged on a GE IN Cell 6000 automated microscope with a 10X objective. Images were collected using 405 and $488 \mathrm{~nm}$ laser lines with DAPI (4',6-diamidino-2-phenylindole) and FITC (fluorescein isothiocyanate) emission filters. Images were analyzed using the GE IN Cell Analyzer Workstation software. Briefly, nuclei were segmented using the Hoechst channel and the cytoplasm was segmented using the GFP channel. For each cell, the mean GFP intensity in each compartment was measured and used to calculate the nuclear to cytoplasmic (N/C) TFEB-GFP ratio. The same method as mentioned above was used to generate the top 30 compounds with highest condensed activity values and robust $Z$-score values.

Isolation and purification of ikarugamycin. SW201073 was extracted from marine-derived bacterium strain SNB-040 isolated from a sediment sample collected from Sweetings Cay, Bahamas. Bacterial spores were collected via a stepwise centrifugation as follows: $2 \mathrm{~g}$ of sediment was dried over $24 \mathrm{~h}$ in an incubator at $35^{\circ}$ $\mathrm{C}$ and the resulting sediment added to $10 \mathrm{~mL} \mathrm{sH}_{2} \mathrm{O}$ containing $0.05 \%$ Tween 20 . After vigorous vortex for $10 \mathrm{~min}$, the sediment was centrifuged at $18,000 \mathrm{rpm}$ for $25 \mathrm{~min}\left(4^{\circ} \mathrm{C}\right)$ and the resulting spore pellet collected. The resuspended spore pellet $\left(4 \mathrm{~mL} \mathrm{sH_{2 }} \mathrm{O}\right)$ was plated on an acidified JMA media, giving rise to individual colonies of SNB-040 after 2 weeks. Analysis of the 16S rRNA sequence of SNB-040 revealed $99 \%$ identity to Streptomyces phaeochromigenes.

Bacterium SNB-040 was cultured in $20 \times 2.8 \mathrm{~L}$ Fernbach flasks each containing $1 \mathrm{~L}$ of seawater-based medium ( $10 \mathrm{~g}$ starch, $4 \mathrm{~g}$ yeast extract, $2 \mathrm{~g}$ peptone, $1 \mathrm{~g}$ $\left.\mathrm{CaCO}_{3}, 40 \mathrm{mg} \mathrm{Fe}_{2}\left(\mathrm{SO}_{4}\right)_{3} \cdot 4 \mathrm{H}_{2} \mathrm{O}, 100 \mathrm{mg} \mathrm{KBr}\right)$ and shaken at $200 \mathrm{rpm}$ at $27^{\circ} \mathrm{C}$. After 7 days of cultivation, sterilized XAD-7-HP resin $\left(20 \mathrm{~g} \mathrm{~L}^{-1}\right)$ was added to absorb the organic products, and the culture and resin were shaken at $200 \mathrm{rpm}$ for $2 \mathrm{~h}$. The resin was filtered through cheesecloth, washed with deionized water, and eluted with acetone. The acetone-soluble fraction was dried in vacuo to yield $4.5 \mathrm{~g}$ of extract.

Crude extract of SNB-040 was fractionated using reverse phase flash column chromatography $(\mathrm{C} 18)$ with a stepwise gradient $(20-100 \%) \mathrm{MeOH} / \mathrm{H}_{2} \mathrm{O}$. Fractions were analyzed by Liquid chromatography-mass spectrometry (LC-MS) using an analytical C18 column and gradient from $10-100 \%$ acetonitrile/water $(0.1 \%$ formic acid) over $17 \mathrm{~min}\left(0.7 \mathrm{~mL} \mathrm{~min}^{-1}\right)$, followed by $100 \%$ acetonitrile for $5 \mathrm{~min}$. Ikarugamycin elutes at $21 \mathrm{~min}$ on this LC-MS method. Fractions containing ikarugamycin were combined, dried, and purified using reverse phase HPLC (phenyl-hexyl column, Phenomenex Luna, $250 \times 10.0 \mathrm{~mm}, 5 \mu \mathrm{m}$ ) at $80 \%$ acetonitrile/water $\left(0.1 \%\right.$ formic acid) and ikarugamycin $t_{\mathrm{R}}=12.5 \mathrm{~min}$ with a strong ultraviolet (UV) absorbance at $254 \mathrm{~nm}$. Ultimately a white amorphous solid (ikarugamycin) with a $\lambda_{\max }$ absorption of 250 and $325 \mathrm{~nm}$ with $\mathrm{m} / z$ [M+H] of 479.2 was purified. ${ }^{1} \mathrm{H}$ NMR $(600 \mathrm{MHz}, \mathrm{DMSO}-\mathrm{d} 6) \delta: 7.69(\mathrm{dd}, J=5.7 \mathrm{~Hz}, 1 \mathrm{H})$, $7.46(\mathrm{~d}, J=15.3 \mathrm{~Hz}, 1 \mathrm{H}), 6.45(\mathrm{br} \mathrm{s}, 1 \mathrm{H}), 6.05\left(\mathrm{dd}, J_{1}=15.3 \mathrm{~Hz}, J_{2}=9.9 \mathrm{~Hz}, 1 \mathrm{H}\right)$, $5.94\left(\mathrm{dt}, J_{1}=15.5 \mathrm{~Hz}, J_{2}=14.4 \mathrm{~Hz}, 1 \mathrm{H}\right), 5.86(\mathrm{~d}, J=9.6 \mathrm{~Hz}, 1 \mathrm{H}), 5.82\left(\mathrm{dd}, J_{1}=14.4\right.$ $\left.\mathrm{Hz}, J_{2}=2.0 \mathrm{~Hz}, 1 \mathrm{H}\right), 5.72\left(\mathrm{dd}, J_{1}=9.6 \mathrm{~Hz}, J_{2}=2.0 \mathrm{~Hz}, 1 \mathrm{H}\right), 3.38(\mathrm{~m}, 1 \mathrm{H}), 3.30(\mathrm{~m}$ $1 \mathrm{H}), 3.28(\mathrm{~m}, 1 \mathrm{H}), 2.49(\mathrm{~m}, 1 \mathrm{H}), 2.39(\mathrm{~m}, 1 \mathrm{H}), 2.25(\mathrm{~m}, 2 \mathrm{H}), 2.10-2.09(\mathrm{~m}, 2 \mathrm{H})$ $2.02(\mathrm{~m}, 1 \mathrm{H}), 1.99(\mathrm{~m}, 1 \mathrm{H}), 1.71(\mathrm{~m}, 1 \mathrm{H}), 1.63(\mathrm{~m}, 1 \mathrm{H}), 1.53(\mathrm{~m}, 1 \mathrm{H}), 1.45(\mathrm{~m}, 1 \mathrm{H})$ 
$1.33-1.31(\mathrm{~m}, 3 \mathrm{H}), 1.26(\mathrm{~m}, 1 \mathrm{H}), 1.16-1.09(\mathrm{~m}, 3 \mathrm{H}), 0.91\left(\mathrm{dd}, J_{1}=8.3 \mathrm{~Hz}, J_{2}=7.2\right.$ $\mathrm{Hz}, 3 \mathrm{H}), 0.86(\mathrm{~d}, J=7.2 \mathrm{~Hz}, 2 \mathrm{H}), 0.66(\mathrm{~m}, 1 \mathrm{H}) .{ }^{13} \mathrm{C}$ NMR $(100 \mathrm{MHz}$, DMSO-d6) $\delta$ : $195.3,181.3,176.6,165.6,140.1,137.8,132.0,130.2,129.1,125.0,101.9,58.4,48.6$, $48.3,47.3,46.7,46.5,42.1,41.1,38.3,37.9,36.9,33.7,27.2,24.8,21.6,21.1,17.7$ 13.1 .

Dose-response assays. Wild-type, GFP-LC3 and GFP-TFEB HeLa cells were seeded in 96-well plates and treated with half log dilutions of compounds in triplicates. Treatment, data acquisition and analysis was identical to that described for the HTS chemical screens. Cathepsin B activity was measured using the Magic $\operatorname{Red}^{\mathrm{TM}}-(\mathrm{RR})_{2}$ Cathepsin B assay kit following a $4 \mathrm{~h}$ treatment with compounds at indicated doses. Raw data was background-corrected, log-transformed and fit with the dose-response function with Graphpad Prism (v6.0) software.

Confocal imaging. All confocal imaging was performed on a Zeiss LSM 700 laser scanning confocal microscope using a 40X/1.3DIC objective. Cells were plated on 4- or 8-well Nunc ${ }^{\mathrm{TM}}$ Lab-Tek $^{\mathrm{TM}}$ II Chambered Coverglass (Thermo Scientific) and allowed to grow for $24 \mathrm{~h}$. After treatment, cells were fixed in $4 \%$ formaldehyde and imaged at excitation wavelengths of $488 \mathrm{~nm}$ (GFP, LysoSensor Green or BODIPY) and $560 \mathrm{~nm}$ (Cy3.5). ImageJ software (NIH) was used to process and analyze the images.

Endosomal maturation rates. HeLa cells were plated on 4- or 8-well $\mathrm{Nunc}^{\mathrm{TM}}$ Lab-Tek ${ }^{\mathrm{TM}}$ II Chambered Coverglass and allowed to grow for $24 \mathrm{~h}$. After $4 \mathrm{~h}$ treatment with compounds or DMSO, cells were incubated with always-ON/OFF$\mathrm{ON} \mathrm{UPS}_{5.3}$ nanoprobes for $5 \mathrm{~min}$ in serum-free medium, then washed three times with PBS before imaging. The $\mathrm{FI}_{\mathrm{OFF}-\mathrm{ON} \text { (BODIPY) }} / \mathrm{FI}_{\text {Always-ON (Cy3.5) }}$ ratio was quantitated with ImageJ. For each cell, a region of interest was defined as the punctae in cytosol that emitted fluorescent signals from both BODIPY and Cy3.5 channels. Fluorescent intensity ratio was calculated for each intracellular punctate as $R=\left(F_{1}-B_{1}\right) /\left(F_{2}-B_{2}\right)$ where $F_{1}$ and $F_{2}$ are the fluorescence intensities from BODIPY and Cy3.5 channels respectively, and $B_{1}$ and $B_{2}$ are the corresponding background values determined from a region on the same images that was near the punctae in the cytosol. All the ratios of each nanoprobe were normalized to their end time-point ratio, and the curves were fit with Graphpad Prism (v6.0) software.

Fura-2 $\mathrm{Ca}^{2+}$ imaging. HeLa cells treated with compounds or DMSO for $4 \mathrm{~h}$ were loaded with Fura-2-AM $(3 \mu \mathrm{M})$ in cell culture medium for $60 \mathrm{~min}$ at $37^{\circ} \mathrm{C}$. Cells were washed twice then incubated in fresh medium for $30 \mathrm{~min}$ to allow complete de-esterification of intracellular AM esters. Imaging was performed at $37^{\circ} \mathrm{C}$ with $5 \% \mathrm{CO}_{2}$ on an epifluorescent microscope (Deltavision, Applied Precision) equipped with a digital monochrome Coolsnap HQ2 camera (Roper Scientific, Tucson, AZ). Fluorescence images were collected using SoftWoRx v3.4.5 (Universal Imaging, Downingtown, PA). Data were recorded at excitation/emission wavelengths of $340 / 510 \mathrm{~nm}$ (Fura-340 filter) and $387 / 510 \mathrm{~nm}$ (Fura-380 filter). The single band pass excitation filter for Fura-340 and Fura-380 is 26 and $11 \mathrm{~nm}$, respectively, and the band pass of emission filters for Fura-340 and Fura-380 is $84 \mathrm{~nm}$. Intracellular calibration of Fura- 2 was accomplished by manipulating the $\mathrm{Ca}^{2+}$ levels inside cells using the ionphore ionomycin $(20 \mu \mathrm{M}$, Sigma-Aldrich) and by incubating cells in buffers with various $\mathrm{Ca}^{2+}$ concentrations (calcium calibration buffer kit- Invitrogen). Intracellular fluorescence ratios were determined using ImageJ software. Images were background-corrected by subtracting the mean pixel values of a cellfree region near the region of interest. Fluorescent intensity ratio $R=F_{340} / F_{380}$, and $\mathrm{Ca}^{2+}$ concentration can be calculated from Equation 3, where $K_{\mathrm{d}}$ can be obtained from intracellular calibration:

$$
\left[\mathrm{Ca}^{2+}\right]=K_{\mathrm{d}} \times \frac{F_{380, \min }}{F_{380, \text { max }}} \times \frac{\left(R-R_{\min }\right)}{\left(R_{\max }-R\right)}
$$

GFP-TFEB nuclear translocation. GFP-TFEB HeLa cells treated with compounds or DMSO were fixed and stained with Hoechst. Images from at least three different fields per sample were acquired using a $40 \mathrm{X}$ objective on a Zeiss confocal microscope and analyzed with ImageJ. In all, 20-30 cells were evaluated from each image for each sample, and three independent experiments were performed to generate the graphed values.

RNA extraction and qRT-PCR. Total RNA was isolated from MEFs or mouse tissues using RNeasy minipreps (QIAGEN). Complementary DNA (cDNA) was synthesized with the High-capacity RNA-to-cDNA kit (Applied biosystems), and qRT-PCR was performed using TaqMan ${ }^{\circledR}$ Gene Expression Assays (Applied biosystems) for the indicated genes on the LightCycler System (Roche Applied Science). Gapdh was used to normalize RNA input. The mouse probes used in this study were: Tfeb (Mm00448968_m1), Ctsa (Mm00447197_m1), Mcoln 1 (Mm00522550_m1), Ppara (Mm00440939_m1), Ppargcla (Mm01208835_m1), Fgf21 (Mm00840165_g1), and Gapdh (Mm99999915_g1), and the human probes used were TFEB (Hs00292981_m1), CTSA (Hs00264902_m1), MCOLN1 (Hs01100653_m1), PPARGC1A (Hs00173304_m1), PPARA (Hs00947536_m1),
UVRAG (Hs01075434_m1), GAPDH (Hs02758991_g1), and p62/SQSTM1 (Hs01061917_g1). All probes were from Thermo Fisher Scientific Inc.

RNA interference (RNAi). Transfection of siRNA duplexes was used to silence indicated genes. In brief, cells grown in six-well plates were transfected with Lipofectamine ${ }^{\circledR}$ RNAiMAX transfection reagent (Thermo Fisher Scientific) and $100 \mathrm{nM}$ siRNA duplexes targeted against $\mathrm{Na}^{+}-\mathrm{K}^{+}$-ATPase $\alpha_{1}$ subunit (MQ-00611102), PTPMT1 (MQ-029988-02), PPP3CB (MQ-009704-01), MCOLN1 (MQ006281-00), TFEB (MQ-009798-02), and TFE3 (MQ-009363-03, Dharmacon). Treated cells were analyzed $48-72 \mathrm{~h}$ after transfection.

Nanoparticle formulation. AD or IKA (1 mg) together with PEG-PLA polymer ( 9 $\mathrm{mg}$ ) were first dissolved in $1 \mathrm{~mL}$ methanol. The solution was added drop-wise to $10 \mathrm{~mL}$ Milli-Q water. Four to five filtrations through a micro-ultrafiltration system $(<100 \mathrm{kDa}$, Amicon Ultra filter units, Millipore) were used to remove the organic solvent and unencapsulated free drugs. The aqueous solution of UPS nanoparticles was sterilized with a $0.22 \mu \mathrm{m}$ filter unit (Millex-GP syringe filter unit, Millipore). Micelle solutions were then lyophilized and the resulting freeze-dried powder was weighed, dissolved in a mixture of methanol and deionized water $(v / v=9 / 1)$, and analyzed using a Shimadzu UV-1800 UV-Vis spectrophotometer $(\lambda=240 \mathrm{~nm}$, extinction coefficient $=2.0 \times 10^{4} \mathrm{M}^{-1} \mathrm{~cm}^{-1}$ ) to calculate the total amount of micelle encapsulated drug. Nanoparticles were also characterized by dynamic light scattering to evaluate particle size.

Mouse model for in vivo compound delivery. All mouse experiments were approved and carried out following the ethical guidelines established by the Institutional Animal Care and Use Committee at UT Southwestern Medical Center. The investigators were not blinded to allocation during experiments and outcome assessment. Four to 6-weeks-old male C57BL/6 J mice were randomly divided into groups fed a regular diet (Harlan Teklad) or a high-fat diet (HFD) containing 60\% fat (Research Diets). After 1 month, the HFD mice were grouped so that the average body weights of mice in each group were similar. The grouped mice were orally administered DG $\left(2.5 \mathrm{mg} \mathrm{kg}^{-1}\right)$ or its solvent $50 \% \mathrm{HP} \beta \mathrm{CD}$ solution, or intravenously injected with $\mathrm{AD}\left(1 \mathrm{mg} \mathrm{kg}^{-1}\right)$ or IKA $\left(0.5 \mathrm{mg} \mathrm{kg}^{-1}\right)$ encapsulated in the PEG-PLA nanoparticles or empty nanoparticles, three times a week for three weeks. Body weight and food intake were measured twice a week before treatment and every day after treatment in the middle of the light period. The cage tops containing food pellets were weighed, as well as the spilled food in the bottom of the cage. The food intake was corrected for spillage.

In vitro compound release from PEG-PLA micelle. $\mathrm{AD}$ and IKA release from $\mathrm{PEG}_{5000}-\mathrm{PLA}_{5000}$ micelle was measured using a dialysis method. In a typical procedure, $\mathrm{AD}$ or IKA micelle solution $\left(0.5 \mathrm{~mL}, 10 \mathrm{mg} \mathrm{mL}^{-1}\right)$ was added to the upper chamber of a $15 \mathrm{~mL}$ mini dialysis tube $(3.5 \mathrm{k}$ molecular weight cutoff, Fisher Scientific Inc.) with $1 \mathrm{x}$ PBS with $1 \%$ Tween 80 (Sigma-Aldrich). At different time points, $1 \mathrm{~mL}$ solution was removed from the tube and replaced with $1 \mathrm{~mL} 1 \mathrm{x}$ PBS with $1 \%$ Tween 80 . The released AD or IKA was determined by measuring the UV-Vis absorbance of the obtained solution based on the standard curves of AD and IKA. Percentage of compound release was plotted as a function of time to show the release kinetics.

Body composition analysis. At the end of the treatment, the body composition of each mouse was analyzed by EchoMRI (Echo Medical Systems LLC) according to the manufacturer's instructions.

Glucose and insulin tolerance tests. For glucose tolerance tests, the mice were orally administered $1 \mathrm{mg} \mathrm{g}^{-1}$ glucose (Sigma-Aldrich) after a $4 \mathrm{~h}$ fast. For insulin tolerance tests, the mice were intraperitoneally injected with 0.75 milliunit $\mathrm{g}^{-1}$ insulin (Humulin R, Eli Lily) after a $4 \mathrm{~h}$ fast. Blood was drawn from tail veins at indicated time points after injection. Experiments were performed during light period. Serum glucose levels were analyzed using commercial glucose reagents (Sigma).

Serum chemistry analysis. At the end of the treatment, blood was collected from the orbital plexus under anesthesia. Serum was frozen in aliquots and stored at -20 ${ }^{\circ} \mathrm{C}$ for further analysis. Specific enzyme kits were used to detect serum levels of triglyceride (Fisher Scientific), cholesterol (Fisher Scientific) and glucose (SigmaAldrich).

Histology. Livers and other organs were dissected and embedded in OCT. Cryostat sections were cut at $10 \mu \mathrm{m}$. The sections were stored at $-80^{\circ} \mathrm{C}$ and subjected to hematoxylin/eosin and oil red O staining following standard protocol. The immunohistochemistry (IHC) staining of p62 was performed following the protocol of Cell Signaling Technology. The primary antibody was from Abcam, and the SignalStain ${ }^{\circledR}$ boost IHC detection reagent and DAB substrate kit were from Cell Signaling Technology. All the sections were imaged using a NanoZoomer 2.0-HT Digital slide scanner (Hamamatzu) and processed using NDP viewer software. 
Cytotoxicity. HeLa cells were plated in a 96-well plate with a white wall and a clear bottom. After $24 \mathrm{~h}$, cells were treated with various doses of DG, AD, and IKA for 4 h. Cells were then washed with PBS three times, and viability was determined immediately or after $72 \mathrm{~h}$ using CellTiter-Glo ${ }^{\circledR}$ Luminescent Cell Viability Assay (Promega).

HLH-30::GFP nuclear localization assay. Adult TX1941 dal-1(dt2300); sqIs19 [hlh-30p::hlh-30::GFP rol-6(+)] worms were placed on nematode growth medium (NGM) plates with either a test chemical or 5\% DMSO. GFP was scored at various intervals on live worms without mounting using a Zeiss Axio Zoom. V16 fluorescence dissecting microscope equipped with Axiocam 503. No difference was observed between $2 \mathrm{~h}$ and overnight treatment.

C. elegans lifespan analysis. The C. elegans mutant strain, fem-1(hc17ts) IV; dal-1 (dt2300), obtained from the Caenorhabditis Genetics Center at the University of Minnesota, was cultured on NGM plates seeded with the E. coli strain OP50 and consistently maintained at $15^{\circ} \mathrm{C}$. For lifespan assays, a total of 12 age-synchronized nematode adults were transferred to eight replicate NGM plates and grown at $25^{\circ} \mathrm{C}$ to ensure sterility. Age synchronization was achieved through standard hypochlorite treatments. Eggs were placed on NGM plates supplemented with Streptomycin $\left(100 \mu \mathrm{g} \mathrm{m}^{-1}\right)$ and seeded with the E. coli strain, OP50. For compound testing, $200 \mu \mathrm{L}$ of $10 \mu \mathrm{M}$ Ikarugamycin in $5 \%$ DMSO was spotted directly onto OP50 seeded NGM plates. Control plates were prepared by spotting $200 \mu \mathrm{L}$ of $5 \%$ DMSO directly onto OP50 seeded NGM plates. For the first 10 days of adulthood, C. elegans were scored once a day as dead or alive by touch stimulation with a platinum wire. After day 10, animals were scored every other day. Nematodes that crawling off the agar plates were censored from subsequent lifespan analysis. Kaplan-Meier statistical analysis was performed using Prism 7 software. Lifespan experiments were done on two separate occasions.

Statistics. Sample sizes and reproducibility for each figure are denoted in the figure legends. Data were presented as the mean \pm s.d. unless specified. Analysis of variance (ANOVA) approaches were used for comparisons among experimental groups that met the normality distribution assumption. If not, the data was logtransformed or a non-parametric $t$-test was used. One-way ANOVA and two-way ANOVA were used for comparison within groups with single or two variables.

Data availability. The data that support the findings of this study are contained in the figures and the Supplementary Information of this article or are available from the corresponding author upon reasonable request.

Received: 10 November 2016 Accepted: 21 November 2017

Published online: 22 December 2017

\section{References}

1. Yang, L., Li, P., Fu, S., Calay, E. S. \& Hotamisligil, G. S. Defective hepatic autophagy in obesity promotes ER stress and causes insulin resistance. Cell Metab. 11, 467-478 (2010).

2. Singh, R. et al. Autophagy regulates lipid metabolism. Nature 458, 1131-1135 (2009).

3. Ding, W. X. et al. Autophagy reduces acute ethanol-induced hepatotoxicity and steatosis in mice. Gastroenterology 139, 1740-1752 (2010).

4. Hansen, M. et al. A role for autophagy in the extension of lifespan by dietary restriction in C. elegans. PLoS Genet. 4, e24 (2008).

5. Pyo, J.-O. et al. Overexpression of Atg5 in mice activates autophagy and extends lifespan. Nat. Commun. 4, 2300 (2013).

6. Rubinsztein, D. C., Mariño, G. \& Kroemer, G. Autophagy and aging. Cell 146, 682-695 (2011).

7. Settembre, C. et al. TFEB controls cellular lipid metabolism through a starvation-induced autoregulatory loop. Nat. Cell Biol. 15, 647-658 (2013).

8. Lapierre, L. R. et al. The TFEB orthologue HLH-30 regulates autophagy and modulates longevity in Caenorhabditis elegans. Nat. Commun. 4, 2267 (2013).

9. Sardiello, M. et al. A gene network regulating lysosomal biogenesis and function. Science 325, 473-477 (2009).

10. Settembre, C. et al. TFEB links autophagy to lysosomal biogenesis. Science 332, 1429-1433 (2011).

11. Settembre, C. et al. A lysosome-to-nucleus signalling mechanism senses and regulates the lysosome via mTOR and TFEB. EMBO J. 31, 1095-1108 (2012).

12. Medina, D. L. et al. Lysosomal calcium signalling regulates autophagy through calcineurin and TFEB. Nat. Cell Biol. 17, 288-299 (2015).

13. Martina, J. A., Chen, Y., Gucek, M. \& Puertollano, R. MTORC1 functions as a transcriptional regulator of autophagy by preventing nuclear transport of TFEB. Autophagy 8, 903-914 (2012).
14. Carr, C. S. \& Sharp, P. A. A helix-loop-helix protein related to the immunoglobulin E box-binding proteins. Mol. Cell Biol. 10, 4384-4388 (1990).

15. Hodgkinson, C. A. et al. Mutations at the mouse microphthalmia locus are associated with defects in a gene encoding a novel basic-helix-loop-helix-zipper protein. Cell 74, 395-404 (1993).

16. Zhao, G.-Q., Zhao, Q., Zhou, X., Mattei, M. \& De Crombrugghe, B. TFEC, a basic helix-loop-helix protein, forms heterodimers with TFE3 and inhibits TFE3-dependent transcription activation. Mol. Cell. Biol. 13, 4505-4512 (1993).

17. Roczniak-Ferguson, A. et al. The transcription factor TFEB links mTORC1 signaling to transcriptional control of lysosome homeostasis. Sci. Signal. 5, ra42 (2012).

18. Martina, J. A. et al. The nutrient-responsive transcription factor TFE3, promotes autophagy, lysosomal biogenesis, and clearance of cellular debris. Sci. Signal. 7, ra9 (2014).

19. Martina, J. A., Diab, H. I., Brady, O. A. \& Puertollano, R. TFEB and TFE3 are novel components of the integrated stress response. EMBO J. 35, 479-495 (2016).

20. Pastore, N. et al. TFE3 regulates whole-body energy metabolism in cooperation with TFEB. EMBO Mol. Med. 9, 605-621 (2017).

21. Li, Y. et al. Molecular basis of cooperativity in $\mathrm{pH}$-triggered supramolecular self-assembly. Nat. Commun. 7, 13777 (2016).

22. Ma, X. et al. Ultra-pH-sensitive nanoprobe library with broad $\mathrm{pH}$ tunability and fluorescence emissions. J. Am. Chem. Soc. 136, 11085-11092 (2014).

23. Wang, C. et al. A nanobuffer reporter library for fine-scale imaging and perturbation of endocytic organelles. Nat. Commun. 6, 8524 (2015).

24. Kabeya, Y. et al. LC3, a mammalian homologue of yeast Apg8p, is localized in autophagosome membranes after processing. EMBO J. 19, 5720-5728 (2000).

25. Jomon, K., Kuroda, Y., Ajisaka, M. \& Sakai, H. A new antibiotic, ikarugamycin J. Antibiot. 25, 271-280 (1972).

26. Pankiv, S. et al. p62/SQSTM1 binds directly to Atg8/LC3 to facilitate degradation of ubiquitinated protein aggregates by autophagy. J. Biol. Chem. 282, 24131-24145 (2007)

27. Hundeshagen, P., Hamacher-Brady, A., Eils, R. \& Brady, N. R. Concurrent detection of autolysosome formation and lysosomal degradation by flow cytometry in a high-content screen for inducers of autophagy. BMC Biol. 9, 38 (2011).

28. Glynn, I. The action of cardiac glycosides on sodium and potassium movements in human red cells. J. Physiol. 136, 148 (1957).

29. Doughty-Shenton, D. et al. Pharmacological targeting of the mitochondrial phosphatase PTPMT1. J. Pharmacol. Exp. Ther. 333, 584-592 (2010).

30. Mukai, H. et al. FKBP12-FK506 complex inhibits phosphatase activity of two mammalian isoforms of calcineurin irrespective of their substrates or activation mechanisms1. J. Biochem. (Tokyo) 113, 292-298 (1993).

31. Kissinger, C. R. et al. Crystal structures of human calcineurin and the human FKBP12-FK506-calcineurin complex. Nature 378, 641-4 (1995).

32. Hogan, P. G., Chen, L., Nardone, J. \& Rao, A. Transcriptional regulation by calcium, calcineurin, and NFAT. Genes Dev. 17, 2205-2232 (2003).

33. Høyer-Hansen, M. et al. Control of macroautophagy by calcium, calmodulindependent kinase kinase- $\beta$, and Bcl-2. Mol. Cell 25, 193-205 (2007).

34. Sullivan, J. E. et al. Inhibition of lipolysis and lipogenesis in isolated rat adipocytes with AICAR, a cell-permeable activator of AMP-activated protein kinase. FEBS Lett. 353, 33-36 (1994).

35. Zhou, G. et al. Role of AMP-activated protein kinase in mechanism of metformin action. J. Clin. Invest. 108, 1167 (2001).

36. Tokumitsu, $\mathrm{H}$. et al. STO-609, a specific inhibitor of the $\mathrm{Ca}^{2+} /$ calmodulindependent protein kinase kinase. J. Biol. Chem. 277, 15813-15818 (2002).

37. Clapham, D. E. Calcium signaling. Cell 131, 1047-1058 (2007).

38. Berg, T., Strømhaug, P., Løvdal, T., Seglen, P. \& Berg, T. Use of glycyl-Lphenylalanine 2-naphthylamide, a lysosome-disrupting cathepsin $C$ substrate, to distinguish between lysosomes and prelysosomal endocytic vacuoles. Biochem. J. 300, 229-236 (1994).

39. Lytton, J., Westlin, M. \& Hanley, M. R. Thapsigargin inhibits the sarcoplasmic or endoplasmic reticulum Ca-ATPase family of calcium pumps. J. Biol. Chem. 266, 17067-17071 (1991).

40. Zhang, X. et al. MCOLN1 is a ROS sensor in lysosomes that regulates autophagy. Nat. Commun. 7, 12109 (2016).

41. Yuan, Z. et al. Na/K-ATPase tethers phospholipase C and IP3 receptor into a calcium-regulatory complex. Mol. Biol. Cell 16, 4034-4045 (2005).

42. Finck, B. N. \& Kelly, D. P. PGC-1 coactivators: inducible regulators of energy metabolism in health and disease. J. Clin. Invest. 116, 615-622 (2006).

43. Zhang, Y.-N., Poon, W., Tavares, A. J., McGilvray, I. D. \& Chan, W. C. Nanoparticle-liver interactions: Cellular uptake and hepatobiliary elimination. J. Control. Release 240, 332-348 (2016).

44. Klibanov, A. L., Maruyama, K., Torchilin, V. P. \& Huang, L. Amphipathic polyethyleneglycols effectively prolong the circulation time of liposomes. FEBS Lett. 268, 235-237 (1990).

45. Kharitonenkov, A. et al. FGF-21 as a novel metabolic regulator. J. Clin. Invest. $115,1627-1635$ (2005) 
46. Markan, K. R. et al. Circulating FGF21 is liver derived and enhances glucose uptake during refeeding and overfeeding. Diabetes 63, 4057-4063 (2014).

47. Lundåsen, T. et al. PPAR $\alpha$ is a key regulator of hepatic FGF21. Biochem. Biophys. Res. Commun. 360, 437-440 (2007).

48. Renquist, B. J. et al. Melanocortin-3 receptor regulates the normal fasting response. Proc. Natl Acad. Sci. USA 109, E1489-E1498 (2012).

49. Paulson, C. C. Analysis of Caenorhabditis elegans genes required for maintaining intestinal lumen morphology and ingested drug resistance. Southern Methodist University (2009).

50. Miyakawa-Naito, A. et al. Cell signaling microdomain with $\mathrm{Na}$, K-ATPase and inositol 1, 4, 5-trisphosphate receptor generates calcium oscillations. J. Biol. Chem. 278, 50355-50361 (2003).

51. Maron, D. J., Fazio, S. \& Linton, M. F. Current perspectives on statins. Circulation 101, 207-213 (2000)

52. Thomas, C., Pellicciari, R., Pruzanski, M., Auwerx, J. \& Schoonjans, K. Targeting bile-acid signalling for metabolic diseases. Nat. Rev. Drug Discov. 7, 678-693 (2008)

53. Sudhop, T. et al. Inhibition of intestinal cholesterol absorption by ezetimibe in humans. Circulation 106, 1943-1948 (2002).

54. Chang, G.-R. et al. Rapamycin protects against high fat diet-induced obesity in C57BL/6J mice. J. Pharmacol. Sci. 109, 496-503 (2009).

55. Wilkinson, J. E. et al. Rapamycin slows aging in mice. Aging Cell 11, 675-682 (2012).

56. Cabreiro, F. et al. Metformin retards aging in C. elegans by altering microbial folate and methionine metabolism. Cell 153, 228-239 (2013).

57. Giugliano, D. et al. Metformin improves glucose, lipid metabolism, and reduces blood pressure in hypertensive, obese women. Diabetes Care 16, 1387-1390 (1993)

\section{Acknowledgements}

We thank S.M. Ferguson at Yale University, B. Levine, J.B. Brugarolas, and Y. Wan at UT Southwestern Medical Center for generously sharing GFP-TFEB-transfected HeLa cells, GFP-LC3-transfected HeLa cells, p53-/- and p53 ${ }^{-/-}$TSC2 ${ }^{-/-}$MEFs and HepG2 cells; Y. Zhang and D.J. Mangelsdorf at UT Southwestern for helping with the EchoMRI system; M. Gross, J.E. Tomb, and R.A. Brekken at UT Southwestern for helping with high-fatdiet mice; J. Waddle for discussing dal-1(-) C. elegans; L. Ma and D.M. Ramirez and the whole-brain microscopy facility at UT Southwestern Department of Neurology and Neurotherapeutics for helping with tissue sectioning and slide imaging. The WBMF is supported by the Texas Institute for Brain Injury and Repair (TIBIR). We would also like to thank P.E. Scherer, J.M. Cooper, R.M. Valden, B. Eskiocak, and U. Eskiocak for thoughtful comments and discussions. This work is supported by the National Institutes of Health (NIH) grants R01CA192221 (J.G.), R01CA71443 and R01CA176284 (M.A.W.),
Cancer Prevention and Research Institute of Texas (CPRIT) grants RP140140 (J.G.), RP121067, RP110710 (M.A.W.), and the Welch Foundation grant I-1414 (M.A.W.). C W. is a Howard Hughes Medical Institute (HHMI) International Student Research Fellow.

\section{Author contributions}

C.W., M.A.W., and J.G. conceived and designed the project, interpreted the data and wrote the paper. C.W. did all in vitro experiments with the help of S.M. H.N., and B.A.P. helped with the high-throughput screen. R.L., P.M.D., and J.J. designed and performed longevity experiments. Y.L. synthesized UPS $_{4,4}$ polymers for the screen. A.Z. and E.A.M conducted biostatistics analysis for the screen results. N.W.O. and J.B.M. isolated and purified ikarugamycin from SW201073. Z.W., T.Z., Z.L., M.L. G.H., and R.A.B. helped with mouse experiments.

\section{Additional information}

Supplementary Information accompanies this paper at https://doi.org/10.1038/s41467017-02332-3.

Competing interests: The authors declare no competing financial interests.

Reprints and permission information is available online at http://npg.nature.com/ reprintsandpermissions/

Publisher's note: Springer Nature remains neutral with regard to jurisdictional claims in published maps and institutional affiliations.

\section{(c) (i)}

Open Access This article is licensed under a Creative Commons Attribution 4.0 International License, which permits use, sharing, adaptation, distribution and reproduction in any medium or format, as long as you give appropriate credit to the original author(s) and the source, provide a link to the Creative Commons license, and indicate if changes were made. The images or other third party material in this article are included in the article's Creative Commons license, unless indicated otherwise in a credit line to the material. If material is not included in the article's Creative Commons license and your intended use is not permitted by statutory regulation or exceeds the permitted use, you will need to obtain permission directly from the copyright holder. To view a copy of this license, visit http://creativecommons.org/ licenses/by/4.0/.

(C) The Author(s) 2018 